\title{
Allgemeines Verwaltungs- und Verwaltungsprozeßrecht
}

\author{
Inhaltsübersicht
}
A. Einleitung
B. Eckpunkte und Entwicklungslinien nach 1945
I. Allgemeines Verwaltungsrecht
1. Die Entfaltung des rechtsstaatlichen Verwaltungsrechts unter dem Grundgesetz
2. Die Entdeckung der Planung
3. Aufstieg und Fall des Verwaltungsverfahrens
4. Europäisierung und Internationalisierung des Verwaltungsrechts
5. Von der „Krise“ zur „Reform“ des Verwaltungsrechts

II. Verwaltungsprozeßrecht
1. Auf- und Ausbau des verwaltungsgerichtlichen Rechtsschutzsystems in der Nachkriegszeit
2. Rechtsschutzhypertrophie: Die Ausweitung der Klagebefugnis und der gerichtlichen Kontrolldichte
3. Entlastungs- und Beschleunigungsgesetzgebung
4. Europäisierung des Verwaltungsprozesses
5. Gegenwartsprobleme und Funktionswandel der Verwaltungs- gerichtsbarkeit

C. Verarbeitung und Beeinflussung der Rechtsentwicklungen durch juristische Standardwerke

I. Lehrbücher

1. Fundamente

a) Fortführung und Weiterentwicklung des Erbes von Otto Mayer

b) Die Öffnung des Verwaltungsrechts für die Verwaltungswirklichkeit

c) Systematisierung von Einzelbereichen

2. Orientierung im Studium und Rechtsalltag
a) Zwei zentrale Referenzwerke

b) Größere Gesamtdarstellungen

3. Irritationen, Impulse und Sonderwege

II. Kommentare und Handbücher

1. Zwei Pionierwerke der ersten Stunde

a) Die Kommentierung der Verwaltungsgerichtsordnung durch Erich Eyermann und Ludwig Fröhler

b) Die Kommentierung des Verwaltungsverfahrensgesetzes des Bundes durch Ferdinand O. Kopp

2. Die Reaktion auf die Ausdifferenzierung der Verwaltungs(prozeß)rechtsordnung: Großkommentare

3. Reform: Das Projekt der "Grundlagen des Verwaltungsrechts“

D. Folgerungen für das Verhältnis von Rechtswissenschaft und Rechtspraxis 


\section{A. Einleitung}

Verwaltungs- und Verwaltungsprozeßrecht haben unter dem Grundgesetz sowohl in der Praxis als auch der Ausbildung einen ungeahnten Bedeutungszuwachs erfahren. Das dogmatische Fundament des liberalen rechtsstaatlichen Verwaltungsrechts, das bis in die heutige Zeit prägend geblieben ist, stammt aus der zweiten Hälfte des 19. Jahrhunderts. Es beruht im wesentlichen auf den Arbeiten einzelner Wissenschaftler, ${ }^{1}$ unter denen das ,Deutsche Verwaltungsrecht“ von Otto Mayer $^{2}$ herausragt. Um so interessanter erscheint die Frage, welche Rolle die Verwaltungsrechtswissenschaft bei der Fortentwicklung des Verwaltungs- und Verwaltungsprozeßrechts in der Bundesrepublik gespielt hat. ${ }^{3}$ Ihr soll im folgenden am Beispiel der Lehrbuch- und Kommentarliteratur, mithin anhand eines Ausschnitts aus dem Spektrum wissenschaftlicher Publikationsformen ${ }^{4}$ nachgegangen werden. Im Mittelpunkt der Betrachtung stehen die vom Beck-Verlag betreuten Veröffentlichungen; zur Abrundung des Gesamtbildes werden aber auch andere zentrale Werke miteinbezogen. Die Ausführungen gliedern sich in drei Teile: In einem ersten Schritt gilt es, sich einen Überblick über die wesentlichen Eckpunkte der Entwicklung in beiden Rechtsgebieten zu verschaffen (B.), bevor in einem zweiten Schritt Wirkmacht und Einfluß einzelner juristischer Standardwerke näher analysiert werden (C.). Der Beitrag schließt mit einigen allgemeinen Folgerungen zum Verhältnis von Rechtswissenschaft und Rechtspraxis (D.).

${ }^{1}$ Eingehend dazu Stolleis, Geschichte des öffentlichen Rechts in Deutschland, Bd. II, 1992, S. $381 \mathrm{ff}$.

2 Otto Mayer, Deutsches Verwaltungsrecht, 2 Bde., 1895/96, 3. Aufl. 1924. Zur überragenden Bedeutung Otto Mayers vgl. nur Meyer-Hesemann, Die paradigmatische Bedeutung Otto Mayers für die Entwicklung der deutschen Verwaltungsrechtswissenschaft, Rechtstheorie 13 (1982), $496 \mathrm{ff}$.

${ }^{3}$ Diese Frage ist bisher vergleichsweise selten erörtert worden, vgl. aber Ossenbühl, Die Weiterentwicklung der Verwaltungswissenschaft, in: Jeserich/Pohl/von Unruh (Hrsg.), Deutsche Verwaltungsgeschichte, Bd. V, 1987, S. $1143 \mathrm{ff}$.; Stolleis, Verwaltungsrechtswissenschaft in der Bundesrepublik Deutschland, in: Simon (Hrsg.), Rechtswissenschaft in der Bonner Republik, 1994, S. 227 ff.; Schulze-Fielitz, Das Bundesverwaltungsgericht als Impulsgeber für die Fachliteratur, in: FG 50 Jahre Bundesverwaltungsgericht, 2003, S. 1061 ff.; ders., Notizen zur Rolle der Verwaltungsrechtswissenschaft für das Bundesverwaltungsgericht, DV 36 (2003), $421 \mathrm{ff}$;; Bumke, Die Entwicklung der verwaltungsrechtswissenschaftlichen Methodik in der Bundesrepublik Deutschland, in: Schmidt-Aßmann/Hoffmann-Riem (Hrsg.), Methoden der Verwaltungsrechtswissenschaft, 2004, S. $73 \mathrm{ff}$.

${ }^{4}$ Erhellende Analyse zu den verschiedenen wissenschaftlichen Literaturgattungen bei SchulzeFielitz, Was macht die Qualität öffentlich-rechtlicher Forschung aus?, JöR n. F. 50 (2002), 1 (16ff.). 


\section{B. Eckpunkte und Entwicklungslinien nach 1945}

\section{Allgemeines Verwaltungsrecht}

\section{Die Entfaltung des rechtsstaatlichen Verwaltungsrechts unter dem Grundgesetz}

Nach dem Ende des Zweiten Weltkrieges stand das Öffentliche Recht vor vielen neuen Herausforderungen. Als mit Abstand größte sollte sich die Umsetzung und Verarbeitung der Einwirkungen des frisch verabschiedeten Grundgesetzes auf das Verwaltungs- und Verwaltungsprozeßrecht erweisen. Angetrieben durch ein selbstbewußtes und mit höchster Autorität ausgestattetes Bundesverfassungsgericht galt das Interesse von Rechtsprechung und Wissenschaft in den ersten zwei Jahrzehnten der Bundesrepublik vor diesem Hintergrund in erster Linie der rechtsstaatlichen Disziplinierung der Verwaltung. ${ }^{5}$ Zentrale Anliegen waren insoweit die Durchsetzung des Gesetzesvorbehalts in den bislang weitgehend ungeregelten Bereichen des Strafvollzugs, Beamten-, Schul- und Soldatenrechts und anderen Sonderrechtsverhältnissen, ${ }^{6}$ die Ausweitung subjektiver Rechte einschließlich der Stärkung individueller Rechtsschutzmöglichkeiten ${ }^{7}$ die Eingrenzung und Kontrolle administrativer Entscheidungsspielräume, ${ }^{8}$ die Fortentwicklung der Handlungsformenlehre ${ }^{9}$ sowie die Entfaltung des Verfahrensgedankens. ${ }^{10}$

Was die konkrete wissenschaftliche Arbeitsweise angeht, so dominierte ungeachtet der Naturrechtsrenaissance in den 1950er Jahren im Verfassungsrecht von Anfang an die sog. juristische Methode, die sich bereits Mitte des 19. Jahrhunderts in bewußter Abkehr von der additiv-erzählenden und aufgabenbezogenen Stoff-

\footnotetext{
${ }^{5}$ Prägnante Analyse bei Stolleis, Verwaltungsrechtswissenschaft (Fn. 3), S. $227 \mathrm{ff}$. Vgl. ferner zuletzt Wahl, Herausforderungen und Antworten: Das Öffentliche Recht der letzten fünf Jahrzehnte, 2006, S. $31 \mathrm{ff}$.

${ }^{6}$ Grundlegend Jesch, Gesetz und Verwaltung, 1961; Rupp, Grundfragen der heutigen Verwaltungsrechtslehre, 2. Aufl. 1991 (1965), S. $113 \mathrm{ff}$; Ossenbühl, Verwaltungsvorschriften und Grundgesetz, 1968, S. 208 ff., und Krebs, Vorbehalt des Gesetzes und Grundrechte, 1975.

7 Vgl. Lorenz, Der Rechtsschutz des Bürgers und die Rechtsweggarantie, 1973.

8 Vgl. neben dem in Fn. 6 genannten Jesch, Unbestimmter Rechtsbegriff und Ermessen in rechtstheoretischer und verfassungsrechtlicher Sicht, AöR 82 (1957), $163 \mathrm{fff}$; Ehmke, „Ermessen“ und „unbestimmter Rechtsbegriff" im Verwaltungsrecht, 1960; Scholz und Schmidt-Aßmann, Verwaltungsverantwortung und Verwaltungsgerichtsbarkeit, VVDStRL 34 (1976), 145 ff. bzw. $221 \mathrm{ff}$. Die Diskussion ist nachgezeichnet bei Pache, Tatbestandliche Abwägung und Ermessen, 2001.

${ }_{9}^{9}$ Zur Bedeutung der Handlungsformenlehre als zentralem Forschungsgegenstand der Verwaltungsrechtswissenschaft vgl. etwa Krause, Rechtsformen des Verwaltungshandelns, 1974; Ossenbühl, Die Handlungsformen der Verwaltung, JuS 1979, 681 ff.; Schmidt-Aßmann, Die Lehre von den Rechtsformen des Verwaltungshandelns, DVBl. 1989, $533 \mathrm{ff}$., und Pauly, Grundlagen einer Handlungsformenlehre im öffentlichen Recht, in: Becker-Schwarze u.a. (Hrsg.), Wandel der Handlungsformen im öffentlichen Recht, 1991, S. 25 ff.

${ }^{10}$ An wichtigen Arbeiten seien hier nur genannt: Bettermann, Das Verwaltungsverfahren, VVDStRL 17 (1959), $118 \mathrm{ff.}$; Kopp, Verfassungsrecht und Verwaltungsverfahrensrecht, 1971; Wahl, Verwaltungsverfahren zwischen Rechtsschutzauftrag und Verwaltungseffizienz, VVDStRL 41 (1983), 151 ff.; Hill, Das fehlerhafte Verfahren und seine Folgen im Verwaltungsrecht, 1986; Pitschas, Verwaltungsverantwortung und Verwaltungsverfahren, 1990; Hufen, Fehler im Verwaltungsverfahren, 4. Aufl. 2002.
} 
darstellung in der enzyklopädisch ausgerichteten Staatswissenschaft alter Prägung herausgebildet hatte. ${ }^{11}$ Kennzeichnend für die juristische Methode ist zunächst die radikale Einengung des Wahrnehmungsfeldes auf eine rechtsaktbezogene Perspektive. ${ }^{12}$ Die Rechtsordnung besteht danach aus einem Geflecht von geschriebenen oder ungeschriebenen, individuellen oder generellen normativen Aussagen, die unabhängig von den tatsächlichen Aufgaben der Verwaltung ${ }^{13}$ isoliert untersucht werden können. Im Mittelpunkt der Betrachtung stehen insoweit die abschließende staatliche Entscheidung und ihre materielle Rechtswirkung im Verhältnis zwischen Staat und Bürger sowie zwischen einzelnen staatlichen Organen, während der interne Entscheidungsfindungsprozeß und seine Bestimmungsfaktoren (Organisation, Personal, Sachmittel etc.) mit Ausnahme punktueller verfahrensrechtlicher Regelungen nur ganz am Rande in den Blick geraten. ${ }^{14}$ Prägend ist ferner eine systematische Vorgehensweise, die dazu dient, die Fülle des Rechtsstoffes beherrschbar zu machen: ${ }^{15}$ Wiederkehrende Aussagen, Abläufe und Strukturen im positiven Recht und in der Rechtspraxis werden gesammelt, gesichtet und zu Figuren, Begriffen, allgemeinen Instituten und Grundsätzen ausgeformt, nach denen sich der gesamte Rechtsstoff möglichst widerspruchsfrei und überzeugend im Sinne formaler Rationalität ${ }^{16}$ ordnen läßt. Mit der systematischen Vorgehensweise eng verbunden ist die Ausbildung von Rechtsdogmatik, mithin einer Klasse von Sätzen (Definitionen, Prinzipien etc.), die auf das positive Recht und die Rechtsprechung bezogen, aber nicht mit ihrer Beschreibung identisch sind, im Rahmen einer institutionell betriebenen Rechtswissenschaft aufgestellt und diskutiert wer-

11 Allgemein zur juristischen Methode im Verwaltungsrecht vgl. Hueber, Otto Mayer. Die ,juristische Methode“ im Verwaltungsrecht, 1982; Heyen, Otto Mayer. Studien zu den geistigen Grundlagen seiner Verwaltungsrechtswissenschaft, 1981; Ishikawa, Friedrich Franz von Mayer. Begründer der ,,juristischen Methode“ im deutschen Verwaltungsrecht, 1992; Stoerk, Über die juristische Methode, 1996.

12 Klar herausgearbeitet ist dieser Umstand bei Bumke, Relative Rechtswidrigkeit, 2004, S. 12-21, 255-259, sowie ders., Methodik (Fn. 3), S. 75-78. Exemplarisch Winkler, Die Wissenschaft vom Verwaltungsrecht, in: Ermacora u.a. (Hrsg.), Allgemeines Verwaltungsrecht, 1979, S. 3 (9f.): ,,Sich der juristischen ... Methode bedienen, heißt zunächst, alles das und nur das als zum Gegenstand gehörig zu betrachten, was durch Rechtsvorschriften, also durch Normen erfaßt ist, die dem geltenden Recht angehören".

13 Den Aufgaben und Zwecken der Verwaltung wird - wenn überhaupt - lediglich heuristische Funktion beigemessen, repräsentativ insofern Bachof, Die Dogmatik des Verwaltungsrechts vor den Gegenwartsaufgaben der Verwaltung, VVDStRL 30 (1972), 193 (223230).

14 So zutreffend Schmidt-Aßmann, Das Allgemeine Verwaltungsrecht als Ordnungsidee, 2. Aufl. 2004, 4. Kap., Rn. 73-75.

15 Zum Systemdenken im Verwaltungsrecht vgl. aus jüngerer Zeit Schmidt-Aßmann, Ordnungsidee (Fn. 14), 1. Kap., Rn. 2-8, sowie von Danwitz, Verwaltungsrechtliches System und Europäische Integration, 1996, S. 26-86, und Bumke, Relative Rechtswidrigkeit (Fn. 12), S. 2336. Zum Gegenmodell am Beispiel des amerikanischen Common Law vgl. Lepsius, Verwaltungsrecht unter dem Common Law, 1997.

${ }_{16} \mathrm{Zu}$ den unterschiedlichen Formen der Rationalität im rechtswissenschaftlichen Kontext vgl. zuletzt übersichtlich Scherzberg, Rationalität - staatswissenschaftlich betrachtet, in: FS für Hans-Uwe Erichsen, 2004, S. 177 ff., sowie Snellen, (Post-)Modernisierung von Staat und öffentlicher Verwaltung: Die Suche nach Rationalität in der Verwaltungswissenschaft, in: FS Klaus König, 2004, S. $467 \mathrm{ff}$. 
den und normativen Gehalt besitzen. ${ }^{17}$ Rechtsdogmatik ist daher „Systemnutzung und Systembildung zugleich" ${ }^{18}$

\section{Die Entdeckung der Planung}

Die Grenzen der juristischen Methode wurden der Verwaltungsrechtswissenschaft durch das Phänomen staatlicher Planung erstmals nach dem Zweiten Weltkrieg wieder nachhaltig vor Augen geführt. ${ }^{19}$ Zwar war man mit der Aufgabe, die zukünftige Entwicklung des Gemeinwesens nach Maßgabe selbstgesetzter Ziele möglichst weit festzuschreiben, nicht zuletzt aufgrund der planwirtschaftlichen Erfahrungen aus zwei Weltkriegen und ihren Folgen seit längerem vertraut, Anfang der 1960er Jahre setzte aber, getragen durch den damaligen Forschrittsoptimismus, eine regelrechte Planungseuphorie ein. $\mathrm{Zu}$ den bekannten Haushaltsplänen und Bauleitplänen gesellten sich Raumordnungspläne, Regionalpläne, raumbezogene Fachpläne, Entwicklungs- und Bedarfspläne für Schulen, Universitäten, Kindergärten und Krankenhäuser, Stellenpläne, Abfallentsorgungspläne, Bildungspläne, Nahverkehrspläne usw. ${ }^{20}$ Das führte auch zu einer Perspektivenerweiterung innerhalb der verwaltungsrechtlichen Dogmatik: Die herkömmlichen Institute, Prinzipien und Handlungsformen des Verwaltungsrechts orientieren sich am Idealbild der gesetzlich gebundenen Verwaltung und der regulativen Idee der einzig richtigen Entscheidung, die dementsprechend von den Verwaltungsgerichten auch vollständig überprüft werden kann. ${ }^{21}$ Der dazu notwenige Handlungs- und Kontrollmaßstab ist regelmäßig nach dem Wenn (Tatbestand)-Dann (Rechtsfolge)-Schema aufgebaut, das mitunter freilich durch ,sekundäre Elastizitäten“"22 wie z. B. die Einräumung von Beurteilungsspielräumen oder Ermessen wieder relativiert wird. ${ }^{23}$ Solche sog. Konditionalprogramme erklären wenige Ereignisse in der Vergangenheit für entscheidungsrelevant (Tatbestand), entlasten den Entscheider dabei weitgehend von der Reflexion über die möglichen (weiteren) Folgen seiner Entscheidung, die ja bereits im wesentlichen festliegen (Rechtsfolge), und erleichtern auf diese Weise eine sehr

17 Alexy, Theorie der juristischen Argumentation, 3. Aufl. 1996, S. 314. Zur Aufgabe und Funktion der Verwaltungsrechtsdogmatik vgl. ferner Brohm, Die Dogmatik des Verwaltungsrechts vor den Gegenwartsaufgaben in der Verwaltung, VVDStRL 30 (1972), 245 (246-249); ders., Kurzlebigkeit und Langzeitwirkung der Rechtsdogmatik, in: FS für Hartmut Maurer, 2001, S. 1079 (1081 ff.); Schulte, Schlichtes Verwaltungshandeln, 1995, S. 9-13.

18 Schmidt-Aßmann, Ordnungsidee (Fn. 14), 1. Kap., Rn. 5. Winkler, Wissenschaft (Fn. 12), S. $13 \mathrm{ff}$., unterscheidet das ,,konkret-dogmatische Denken“ und das „abstrakt-dogmatische Denken".

19 Zum folgenden weiterführend Bumke, Methodik (Fn. 3), S. 115 f.; Wahl, Herausforderungen (Fn. 5), S. $45 \mathrm{ff}$.

20 Überblick bei Hoppe, Planung, in: Isensee/Kirchhof (Hrsg.), Handbuch des Staatsrechts, Bd. IV, 3. Aufl., \$ 77, Rn. $6 \mathrm{ff}$.

21 Zur These der ,eine[n] richtigen Entscheidung“ als „,fromme[r] Lebenslüge der Verwaltungsgerichtsbarkeit" s. Hans Meyer, Die Kodifikation des Verwaltungsverfahrens und die Sanktion für Verfahrensfehler, NVwZ 1986, 513 (521).

22 Luhmann, Lob der Routine, VerwArch 55 (1964), 1 (12).

23 Betonung dieses Gedankens etwa bei Reiner Schmidt, Flexibilität und Innovationsoffenheit im Bereich der Verwaltungsmaßstäbe, in: Hoffmann-Riem/Schmidt-Aßmann/Schuppert (Hrsg.), Reform des Allgemeinen Verwaltungsrechts, 1993, S. 67 (80). 
weitgehende inhaltliche Kontrolle. ${ }^{24}$ Demgegenüber legen die seit den 1960er Jahren vermehrt auftretenden planungssteuernden Zweckprogramme lediglich die Ziele des Verwaltungshandelns fest und erklären allenfalls einzelne bei der Entscheidung zu berücksichtigenden Belange für erheblich (vgl. z. B. § 1 Abs. 6 BauGB). Der Rechtsanwender muß hier in einem aufwendigen Verfahren verschiedene konfligierende Interessen miteinander abwägen, weitreichende Prognosen für die zukünftige Entwicklung anstellen und selbst Handlungsalternativen entwickeln. Beispielhaft sei hier nur auf die Bauleitplanung, die projektbezogene Fachplanung von Flughäfen, Autobahnen, Abfallentsorgungsanlagen usw. oder Nahverkehrspläne (§ 8 Abs. 3 PBefG) hingewiesen.

Es liegt auf der Hand, daß weder die verwaltungsrechtliche Praxis noch die Verwaltungsrechtswissenschaft auf diese Herausforderungen sofort adäquat reagieren konnte. Deshalb suchte man zunächst das Gespräch mit anderen Disziplinen, wie es z.B. in den sechs von Joseph H. Kaiser herausgegebenen Bänden zum Thema „Planung“ dokumentiert ist. ${ }^{25}$ Dort finden sich neben vertrauten Ausführungen zu den „Rechtsfragen der Wirtschaftsplanung“ oder den ,,verfassungsrechtlichen Problemen einer zentralen staatlichen Planung“ auch Beiträge zur Planung aus ökonomischer, verwaltungs- oder militärwissenschaftlicher Sicht sowie Darstellungen zu den verschiedensten Bereichen politischer Planung. Es sollte jedoch zuvörderst Aufgabe des BVerwG bleiben, die Umstellung des Verwaltungsrechts von der herkömmlichen Konditionalprogrammierung in eine Finalprogrammierung juristisch zu verarbeiten und über mehrere Jahrzehnte hinweg eine ausdifferenzierte eigenständige Dogmatik des Planungsrechts zu entwickeln. ${ }^{26}$

\section{Aufstieg und Fall des Verwaltungsverfahrens}

Seit den fünfziger Jahren bemüht man sich um eine angemessene gesetzliche Ausgestaltung des Verwaltungsverfahrens. Dabei lassen sich verschiedene Phasen der Diskussion unterscheiden: In der ersten Phase ging es um die Kodifikation der bis dahin lediglich gewohnheitsrechtlich anerkannten Verfahrensregelungen. Sie endete nach zähem Ringen mit der Verabschiedung des Verwaltungsverfahrensgesetzes des Bundes am 15. Januar 1976, ${ }^{27}$ dem die meisten Bundesländer weitgehend in-

24 Grundlegend zum Unterschied zwischen Konditional- und Zweckprogrammen Luhmann, Funktionale Methode und juristische Entscheidung, AöR 94 (1969), 1 ff. Zur zentralen Bedeutung der konditionalen Normstruktur für das deutsche Verwaltungsrecht vgl. zuletzt wieder Breuer, Zur Lage der deutschen Verwaltungsrechtswissenschaft, DV 36 (2003), 271 (275ff.).

25 Aus verwaltungsrechtlicher Sicht sind ferner hervorzuheben: Wahl, Rechtsfragen der Landesplanung und Landesentwicklung, 2 Bde. 1978; Ernst/Hoppe, Das öffentliche Bau- und Bodenrecht, Raumplanungsrecht, 1978 (weitergeführt als Hoppe/Bönker/Grotefels, Öffentliches Baurecht, 2. Aufl. 2002).

26 Den Anfang machten BVerwGE 34, $301 \mathrm{ff}$; 45, $309 \mathrm{ff}$. Schulze-Fielitz, Bundesverwaltungsgericht (Fn. 3), S. 1065, spricht zu Recht von einem ,,innovativen Glanzstück“ in der Judikatur. Rückblickend statt vieler Hoppe, Entwicklung von Grundstrukturen des Planungsrechts durch das BVerwG, DVB1. 2003, 697ff. m.w. N. Vgl. ferner die Beiträge in den Festschriften für Schlichter (1995), Blümel (1999) und Hoppe (2000), hier: insbes. $975 \mathrm{ff}$.

27 Gesetz vom 25. 5. 1976 (BGBl. I S. 1253). 


\section{Allgemeines Verwaltungsrecht}

haltsgleiche Verwaltungsverfahrensgesetze folgen ließen. ${ }^{28}$ Parallel wurden die Abgabenordnung $^{29}$ und der Allgemeine Teil des Sozialgesetzbuches ${ }^{30}$ erlassen (DreiSäulen-Modell). Die zweite Phase ${ }^{31}$ war gekennzeichnet durch eine breite ,,Verfahrenseuphorie“"32 und den Aufstieg des Verfahrensgedankens zum neuen „Paradigma“ der Rechtswissenschaft. ${ }^{33}$ Es setzte sich die Erkenntnis durch, daß dem Verwaltungsverfahren als „Verwirklichungsmodus“ des materiellen Verwaltungsrechts $^{34}$ eine zentrale Bedeutung für die Erfüllung des Funktionsauftrags der Verwaltung zukommt. ${ }^{35}$ Dieser Funktionsauftrag erschöpft sich nicht in der Rechtmäßigkeit des Verwaltungshandelns, sondern ist gleichermaßen gerichtet auf gemeinwohlorientierte, sachlich zweckmäßige, bürgernahe und zukunftstaugliche Maßnahmen ${ }^{36}$ und deren Akzeptanz. ${ }^{37}$ In dem Maße, in dem es aufgrund der Komplexität der Lebenssachverhalte immer schwieriger wird, durch inhaltliche Direktiven das Entscheidungsergebnis zu bestimmen, erlangt das Entscheidungsverfahren zunehmend größeres Gewicht. Das zeigt sich besonders anschaulich im Planungsrecht, zunehmend aber auch in anderen Rechtsbereichen, etwa bei der Verteilung knapper Güter in Konkurrenzsituationen (z.B. Vergabe von Studienplätzen, Zuweisung von Start- und Landerechten auf Flughäfen, Besetzung von Beamtenstellen) oder Entscheidungen unter Unsicherheitsbedingungen, wie sie etwa für das Atom-, Arzneimittel- oder Gentechnikrecht kennzeichnend sind. ${ }^{38}$

28 Übersichtlich Stelkens, in: Stelkens/Bonk/Sachs, VwVfG, 6. Aufl. 2001, Einleitung, Rn. 9 ff. Vgl. ferner Ule, in: Jeserich/Pohl/von Unruh (Hrsg.), Deutsche Verwaltungsgeschichte, Bd. V, 1987, S. 1162 ff. Freilich ist der Vereinheitlichungsprozeß nicht abgeschlossen, weiterführend etwa Wahl, Neues Verfahrensrecht für Planfeststellung und Anlagengenehmigung - Vereinheitlichung des Verwaltungsverfahrens oder bereichsspezifische Sonderordnung?, in: Blümel/Pitschas (Hrsg.), Reform des Verwaltungsverfahrensrechts, 1994, S. 83 ff. Allgemein zur Bedeutung der Kodifikationsidee für das Verwaltungsrecht vgl. auch Voßkuhle, Kodifikation als Prozeß, in: Schlosser (Hrsg.), Das Bürgerliche Gesetzbuch 1896-1996, 1997, S. 77 ff.; Kahl, Das Verwaltungsverfahrensgesetz zwischen Kodifikationsidee und Sonderrechtsentwicklungen, in: Hoffmann-Riem/Schmidt-Aßmann (Hrsg.), Verwaltungsverfahren und Verwaltungsverfahrensgesetz, 2002, S. $67 \mathrm{ff}$.

29 Abgabenordnung vom 16. 3. 1976 (BGBl. I S. 613).

30 Allgemeiner Teil des Sozialgesetzbuches vom 11. 12. 1975 (BGBl. I S. 3015).

31 Zur Bilanz nach 10 Jahren vgl. Bonk, Vereinheitlichung und Fortentwicklung des Verwaltungsverfahrensrechts in Bund und Ländern, DVBl. 1986, $485 \mathrm{ff}$.; Stelkens, Bereinigung des Verwaltungsverfahrensrechts, NVwZ 1986, $541 \mathrm{ff}$. Zur Bilanz nach 15 Jahren Schoch, Der Verfahrensgedanke im Allgemeinen Verwaltungsrecht, DV 25 (1992), $21 \mathrm{ff}$.

32 Ossenbühl, Kernenergie im Spiegel des Verfassungsrechts, DÖV 1981, 1 (6).

33 So Hill, Verfahren (Fn. 10), S. $193 \mathrm{ff}$. Allgemein zur Bedeutung des Verfahrensgedankens im Öffentlichen Recht vgl. ferner nur Schmidt-Aßmann, in: Lerche/Schmitt Glaeser/SchmidtAßmann, Verfahren als staats- und verwaltungsrechtliche Kategorie, 1984, S. $1 \mathrm{ff}$.

34 Begriffsprägend Wahl, VVDStRL 41 (1983), 141 (153).

35 Zutreffend Laubinger, Der Verfahrensgedanke im Verwaltungsrecht, in: König/Merten (Hrsg.), Verfahrensrecht in Verwaltung und Verwaltungsgerichtsbarkeit, 2000, S. 47 (55). Zu weiteren Funktionen des Verwaltungsverfahrens anschaulich Schuppert, Verwaltungswissenschaft, 2000, S. 794 ff. m.w. N.

36 Ausführlich statt vieler Hill, Verfahren (Fn. 10), S. $199 \mathrm{ff}$.

37 Dazu nur Würtenberger, Die Akzeptanz von Verwaltungsentscheidungen, 1996, S. $98 \mathrm{ff}$.

$38 \mathrm{Zu}$ den ,Strukturen und Bauformen neuer Verwaltungsverfahren“ vgl. den gleichnamigen Beitrag von Voßkuhle, in: Hoffmann-Riem/Schmidt-Aßmann (Hrsg.), Verwaltungsverfahren (Fn. 28), S. $277 \mathrm{ff}$. 
Gleichwohl hat der Gesetzgeber seit Anfang der 1990er Jahre entgegen dem Trend auf europäischer Ebene ${ }^{39}$ und trotz heftiger Kritik aus der Wissenschaft ${ }^{40}$ eine dritte Phase eingeläutet und im Rahmen der sog. Beschleunigungsgesetzgebung ${ }^{41}$ tiefgreifende Einschnitte an den bestehenden Strukturen des Verwaltungsverfahrens vorgenommen. Der Reigen der verabschiedeten Neuerungen reicht von vielfältigen Verfahrenserleichterungen, insbesondere bei Genehmigungs- ${ }^{42}$ und Massenverfahren $^{43}$ über die Einführung neuer, teilweise optional wählbarer Verfahrenstypen $^{44}$ (Plangenehmigung, ${ }^{45}$ Anzeige- und Genehmigungsfreistellungsverfahren ${ }^{46}$ ) bis hin zur Ausweitung von Unbeachtlichkeits-, ${ }^{47}$ Heilungs- ${ }^{48}$ und Präklusionsvorschriften. ${ }^{49}$ Vorrangiges Ziel dieser Regelungen ist die „nachfragegerechte“

39 Zum prozeduralen Steuerungsanliegen des Verwaltungsverfahrensrechts aus europäischer Sicht statt vieler Schmidt-Aßmann/Ladenburger, Umweltverfahrensrecht, in: Rengeling (Hrsg.), Handbuch zum Europäischen und Deutschen Umweltrecht, Bd. 1, 2. Aufl. 2003, § 18, Rn. $1 \mathrm{ff}$.

40 Statt vieler vgl. Sparwasser, Das Genehmigungsverfahrensbeschleunigungsgesetz, AnwBl. 2000, 658 ff.; Voßkuhle, Ökonomisierung des Verwaltungsverfahrens, DV 34 (2001), 347 (349ff.) m. w. N.

41 Wichtige Etappen stellen hier das Verkehrswegeplanungsgesetz vom 16. 12. 1991 (BGBl. I S. 2174), das Investitionserleichterungs- und Wohnbaulandgesetz vom 22. 4. 1993 (BGBl. I S. 466), das Planungsvereinfachungsgesetz vom 17. 12. 1993 (BGB1. I S. 2123), das Gesetz zur Änderung verwaltungsverfahrensrechtlicher Vorschriften vom 2. 5. 1996 (BGB1. I S. 656), das Gesetz zur Beschleunigung von Genehmigungsverfahren (GenBeschlG) vom 12. 9.1996 (BGBl. I S. 1354), das Gesetz zur Beschleunigung und Vereinfachung immissionsschutzrechtlicher Genehmigungsverfahren vom 15. 10. 1996 (BGBl. I S. 1498), das 6. VwGO-Änderungsgesetz vom 1. 11. 1996 (BGBl. I S. 1626) und das Zweite Gesetz zur Änderung verwaltungsverfahrensrechtlicher Vorschriften vom 6. 8. 1998 (BGBl. I S. 2022) dar. Vgl. dazu die informative Übersicht bei Schmidt-Kötters, Verfahrensprivatisierung als Element innovativer Gesetzgebungspolitik, in: Hoffmann-Riem/Schneider (Hrsg.), Verfahrensprivatisierung im Umweltrecht, 1996, S. 31 (35ff.); Püttner/Guckelberger, Beschleunigung von Verwaltungsverfahren, JuS 2001, 218 ff., jeweils m. w. N.

42 Vgl. § 71 b VwVfG (Zügigkeitsgebot), § 71c VwVfG (Beratungs- und Auskunftspflicht), $\S 71 \mathrm{~d}$ VwVfG (Sternverfahren), § 71 e VwVfG (Antragskonferenz).

43 Die Richtzahl von Massenverfahren ist von 3000 auf 50 Personen vermindert worden (\$§ 17 Abs. 4 S. 2, 67 Abs. 1 S. 4, 69 Abs. 2 S. 2 und Abs. 3 S. 2,73 Abs. 5 S. 2 Nr. 4 und Abs. 6 S. 4, 74 Abs. 5 S. 1 VwVfG). Die Bekanntgabe durch Einzelbenachrichtigung kann somit häufiger durch öffentliche Bekanntgabe ersetzt werden, § 41 Abs. 3 S. 1 VwVfG.

$44 \mathrm{Vgl}$. den Überblick bei Schmitz, Moderner Staat - Modernes Verwaltungsverfahrensrecht, NVwZ 2000, 1238 (1239f.).

45 Vgl. $\$ 74$ Abs. 6 VwVfG.

46 Zum Genehmigungsfreistellungsverfahren und zum Anzeige- bzw. Kenntnisgabeverfahren in den einzelnen Landesbauordnungen vgl. Korioth, Der Abschied von der Baugenehmigung nach § 67 BauO NW 1995 - Gelungene Vereinfachung und Beschleunigung des Bauens von Wohngebäuden?, DÖV 1996, 665 ff.; Erbguth/Stollmann, Das bauordnungsrechtliche Freistellungsverfahren, BayVBl. 1996, 65 ff.; Fluck, Änderungen genehmigungsbedürftiger Anlagen nach $\S \S 15,16$ BImSchG i.d.F. der Beschleunigungsnovelle, VerwArch 88 (1997), 265 (283 ff.).

47 Vgl. $\$$ 46, 75 Abs. 1 a S. 1 VwVfG.

48 Vgl. $\$ \S 45$ Abs. 2, 75 Abs. 1 a S. 2 VwVfG; $\$ 87$ Abs. 1 S. 2 Nr. 7, 114 S. 2 VwGO.

49 Vgl. insbes. $§ 71$ d Abs. 2, §73 Abs. 3 a, Abs. 4 S. 3, Abs. 6 VwVfG. Zu den unterschiedlichen Präklusionskategorien vgl. Brandt, Präklusion im Verwaltungsverfahren, NVwZ 1997, 233 ff. Krit. zum Beschleunigungseffekt Danwitz, Umweltrechtliche Präklusionsnormen zwischen Verwaltungseffizienz und Rechtsschutzgarantie, UPR 1996, 323 ff.; Solveen, Zur materiellen Präklusion im Fernstraßenrecht, DVBl. 1997, 803 (804ff., 809). Grundsätzlich zur Problematik Röhl/Ladenburger, Die materielle Präklusion im raumbezogenen Verwaltungsrecht, 1997. 
Beschleunigung von Genehmigungs- und Planungsverfahren. ${ }^{50}$ Der schwerfällige Rhythmus der Verwaltung soll zur Stärkung des Standorts Deutschland dem der global gewordenen Weltwirtschaft angepaßt werden, die rasch und flexibel auf die Signale des Marktes reagieren muß. ${ }^{51} \mathrm{Ob}$ die Abwertung des Verfahrensgedankens bei gleichzeitiger Aufrechterhaltung einer hohen materiellen Prüfungsdichte hier der richtige Weg ist, wird man indes nachhaltig bezweifeln müssen.

\section{Europäisierung und Internationalisierung des Verwaltungsrechts}

Weitreichende Wandlungs- und Modernisierungsimpulse empfangen das Verfassungs- ${ }^{52}$ und Verwaltungsrecht ${ }^{53}$ ferner zunehmend durch den Prozeß der Europäisierung und Internationalisierung. Insgesamt gesehen haben das Europarecht und das moderne Völkerrecht eine „,zweite Phase des Öffentlichen Rechts“ in Deutschland eingeläutet. ${ }^{54}$ Vor allem die ,qualitativ-strukturellen“ ${ }^{55}$ Einschnitte des Gemeinschaftsrechts in das nationale Verwaltungsrecht wurden anfänglich von einem Teil der Literatur geradezu als ,,rechtsmißbräuchlich und besatzungsähnlich“ empfunden. ${ }^{56}$ Besonders heftige Kontroversen löste etwa die Reduzierung

${ }^{50}$ Vgl. BT-Drs. 13/445, S. 1, 6; BT-Drs. 13/3993, S. 1; BT-Drs. 13/3995, S. 1; BT-Drs. 13/ 3996, S. 1. Wegweisend war der Bericht der Unabhängigen Expertenkommission zur Vereinfachung und Beschleunigung von Planungs- und Genehmigungsverfahren, Bundesministerium für Wirtschaft (Hrsg.), Investitionsförderung durch flexible Genehmigungsverfahren, 1994, Rn. $207 \mathrm{ff}$.

51 Grundlegend aus historischer und rechtsvergleichender Sicht zur Bedeutung des Zeitelements im Genehmigungsverfahren Bullinger, Verwaltung im Rhythmus von Wirtschaft und Gesellschaft, JZ 1991, $53 \mathrm{ff}$. Vgl. ferner Brohm, Beschleunigung der Verwaltungsverfahren, NVwZ 1991, $1025 \mathrm{ff}$., sowie ausfuhrlich Rombach, Der Faktor Zeit in umweltrechtlichen Genehmigungsverfahren, 1994, S. 147 ff. m.w. N.

52 Vgl. Huber, Europäisches und nationales Verfassungsrecht, VVDStRL 60 (2001), 194 (222ff.); Di Fabio, Der Verfassungsstaat in der Weltgesellschaft, 2001; Tietje, Die Staatsrechtslehre und die Veränderungen ihres Gegenstandes: Konsequenzen von Europäisierung und Internationalisierung, DVBl. 2003, 1081 ff.; Bryde, Konstitutionalisierung des Völkerrechts und Internationalisierung des Verfassungsrechts, Der Staat 42 (2003), 61 ff.; Giegerich, Europäische Verfassung und deutsche Verfassung im transnationalen Konstitutionalisierungsprozeß: wechselseitige Rezeption, konstitutionelle Evolution und föderale Verflechtung, 2003; Ruffert, Die Globalisierung als Herausforderung an das Öffentliche Recht, 2004.

53 Übersichtlich statt vieler Schoch, Die Europäisierung des Allgemeinen Verwaltungsrechts und der Verwaltungsrechtswissenschaft, DV Beiheft 2 (1999), 135 ff.; Schmidt-Aßmann, Ordnungsidee (Fn. 14), 1. Kap., Rn. 50-67. Vgl. ferner die Staatsrechtslehrerreferate von Zuleeg und Rengeling, Deutsches und europäisches Verwaltungsrecht - wechselseitige Einwirkungen, VVDStRL 53 (1994), 154 ff. bzw. $202 \mathrm{ff}$., sowie die vier „,im Nachgang“ erschienenen Habilitationsschriften von v. Danwitz, Verwaltungsrechtliches System (Fn. 15); Brenner, Der Gestaltungsauftrag der Verwaltung in der Europäischen Union, 1996; Hatje, Die gemeinschaftsrechtliche Steuerung der Wirtschaftsverwaltung, 1998, und Kadelbach, Allgemeines Verwaltungsrecht unter europäischem Einfluß, 1999. Zur Internationalisierung vgl. ferner z. B. Tietje, Internationalisiertes Verwaltungshandeln, 2001; König, Öffentliche Verwaltung und Globalisierung, VerwArch 92 (2001), $475 \mathrm{ff}$.

${ }^{54}$ So Wahl, Zwei Phasen des Öffentlichen Rechts nach 1949, in: ders., Verfassungsstaat, Europäisierung, Internationalisierung, 2003, S. 411 (422 ff.).

55 Schoch, Die Europäisierung des Allgemeinen Verwaltungsrechts, JZ 1995, 109 (111).

${ }^{56}$ So Salzwedel/Reinhardt, Neuere Tendenzen im Wasserrecht, NVwZ 1991, 946 (947). Vgl. auch Dünchheim, Die Europäisierung der Verwaltungsrechtsordnung und die „Hydra des Europäischen Rechts“, VR 1996, 181 (186); Scholz, Zum Verhältnis von europäischem Gemein- 
des Vertrauensschutzes bei der Rücknahme gemeinschaftswidriger Subventionen, ${ }^{57}$ die Verwerfungskompetenz deutscher Verwaltungsbehörden in bezug auf gemeinschaftswidrige Vorschriften des deutschen Rechts, ${ }^{58}$ die Erweiterung der Staatshaftung für normatives Unrecht ${ }^{59}$ oder die Verabschiedung der Verwaltungsvorschrift als taugliches Instrument der Rechtskonkretisierung ${ }^{60}$ aus. Mittlerweile haben sich die Wogen hier aber wieder etwas geglättet.

Indes darf die Europäisierung des Verwaltungsrechts nicht auf die Neuinterpretationen und Inhaltsänderungen gemeinschaftsrechtlich determinierter nationaler Regelungen und Rechtsinstitute beschränkt werden. Die einzelnen Staaten, und mit ihnen die Bundesrepublik, sind vielmehr gezwungen, völlig neue Instrumente, Regelungstypen und Konzepte mit weitreichenden Folgewirkungen in die eigene Rechtsordnung $\mathrm{zu}$ integrieren. Man denke nur an die UmweltinformationsRichtlinie, ${ }^{61}$ die Managementkonzepte des Umweltaudit, die Zertifizierungs- und Akkreditierungsmodelle im Produktsicherheitsrecht ${ }^{62}$ oder etwa die Einführung von Umweltzertifikaten. ${ }^{63}$ Gleichzeitig steigt der Druck, im internationalen Wettbewerb der Rechtssysteme ${ }^{64}$ selbst Vorschläge $\mathrm{zu}$ formulieren und in den Rechtsetzungsprozeß auf europäischer und internationaler Ebene bereits im Vorfeld einzuspeisen.

Diese neuen Herausforderungen haben auch Konsequenzen für die Tätigkeitsfelder, das Selbstverständnis und die Arbeitsweise des (Verwaltungs-)Rechtswissenschaftlers. ${ }^{65}$ Denn allein mit den herkömmlichen Methoden einer auf die systematische Auslegung von Norm-Texten und Sachverhalten spezialisierten Verstehenswissenschaft, ${ }^{66}$ die am Tätigkeitsbild des Richters orientiert ist, lassen sich die Anforderungen und Zumutungen der Europäisierung und Internationalisie-

schaftsrecht und nationalem Verwaltungsverfahrensrecht, DÖV 1998, $261 \mathrm{ff}$. , und zuletzt wieder Breuer, Verwaltungsrechtswissenschaft (Fn. 24), $283 \mathrm{ff}$.

${ }^{57}$ EuGH, NVwZ 1990, 1161 (BUG-Alutechnik); EuZW 1997, 277 ff. (Alcan II).

${ }^{58}$ In diese Richtung gehend EuGH, NJW 1978, 1741 f.; EuGHE 1989, 1839 (1870f.). Eingehend dazu Jamrath, Normenkontrolle der Verwaltung und euröpäisches Gemeinschaftsrecht, 1993, S. $77 \mathrm{ff} ., 120 \mathrm{ff}$.

${ }^{9}$ EuGH, NJW 1996, 1267 (Brasserie du Pêcheur); NJW 1996, 3141 (Dillenkofer); EuZW 1996, 274 ff. (British Telecom); EuZW 1996, 435 ff. (Hedley Lomas).

${ }^{60} \mathrm{EuGH}, \mathrm{NVwZ} 1991,866 \mathrm{ff}$. (TA-Luft).

${ }^{61}$ Richtlinie des Rates 90/313/EWG vom 7. 6. 1990 über den freien Zugang zu Informationen über die Umwelt, ABl. EG 1990, Nr. L 18, S. 56.

62 Scheel, Privater Sachverstand im Verwaltungsvollzug des europäischen Rechts, 1999, S. 33 ff.; Röhl, Akkreditierung und Zertifizierung im Produktsicherheitsrecht, 2000.

${ }^{63}$ Vgl. Richtlinie 2003/87/EG des Europäischen Parlaments und des Rates vom 13. 10. 2003 über ein System für den Handel mit Treibhausgasemissionszertifikaten in der Gemeinschaft und zur Änderung der Richtlinie 96/61/EG des Rates, ABl. EU L 275, S. 32.

${ }^{64}$ Zum Systemwettbewerb der Rechtsordnungen vgl. z. B. Vollmöller, Die Globalisierung des öffentlichen Wirtschaftsrechts, 2001, S. 177 ff. m.w. N. Krit. Dreher, Wettbewerb oder Vereinheitlichung der Rechtsordnungen in Europa?, JZ 1999, 105 (109).

${ }^{65}$ Vgl. nur Schmidt-Aßmann, Zur Situation der rechtswissenschaftlichen Forschung, JZ 1995, 2 (9f.); v. Bogdandy, Beobachtungen zur Wissenschaft vom Europarecht, Der Staat 40 (2001), 3-9, 40-43 m. w. N.; Häberle, Der europäische Jurist, JöR 50 n. F. (2002), 123 ff.; Ruffert, Die Europäisierung der Verwaltungsrechtslehre, DV 36 (2003), $293 \mathrm{ff.}$

${ }^{66}$ Zur Jurisprudenz als „,verstehender“ Wissenschaft vgl. statt vieler Larenz/Canaris, Methodenlehre der Rechtswissenschaft, 3. Aufl. 1995, S. $25 \mathrm{ff}$. 
rung sowie der daraus resultierende Reformbedarf kaum bewältigen. Die Überlagerung und Verschleifung mehrerer, meistens wenig aufeinander abgestimmter Normebenen sowie der offene Finalitätscharakter vieler gemeinschafts- und internationalrechtlicher Vorgaben führt trotz Anschwellens der Regelungsmasse und partieller Verrechtlichungstendenzen zu einer Ausdünnung des erkenntnisleitenden dogmatischen Geflechts. Bevor Rechtsregeln, Figuren, Institute und Lehrsätze neu ausgeformt werden können, stellt sich in der Regel zunächst die vorgelagerte Frage, welche rechtlichen und organisatorischen Rahmenbedingungen überhaupt erfüllt sein müssen, damit hinreichend legitimierte Politikvorstellungen verwirklicht und bei möglichst sparsamem Ressourceneinsatz sachrichtige, gemeinwohlfördernde Entscheidungen produziert werden können, die auch realistische Vollzugschancen besitzen. Ohne geschichts- und theoriegeleitetes Denken, ohne ausreichende Kenntnis der einzelnen Realbereiche und ohne einen problemorientierten grenzüberschreitenden Austausch von rechtlichen Argumenten, Lösungsansätzen und Erfahrungen wird man diese Frage kaum beantworten können. Die gegenwärtige Aufgabe des (Verwaltungs-)Rechtswissenschaftlers besteht daher immer häufiger darin, innerhalb eines veränderbaren und sich stetig verändernden rechtlichen Rahmens mögliche Handlungsalternativen aufzuzeigen, ihre Folgen abzuschätzen, Interessengegensätze offenzulegen und rational begründete, praktische Entscheidungsvorschläge $\mathrm{zu}$ erarbeiten, die je nach Brauchbarkeit dann ihrerseits wieder rückgebunden werden können an den dogmatischen Diskurs. ${ }^{67}$ Sicherlich ist diese Aufgabe nicht völlig neu, besaßen doch Rechtsfortbildung und Rechtspolitik ebenso wie die Rechtsvergleichung ${ }^{68}$ immer schon einen wichtigen Stellenwert innerhalb der Verwaltungsrechtswissenschaft. ${ }^{69}$ Alle drei Bereiche haben aber unter dem Einfluß der Europäisierung und Internationalisierung offensichtlich eine neue quantitative und qualitative Dimension erreicht. ${ }^{70}$

67 Eine Schwerpunktverlagerung der Forschungstätigkeit von einer anwendungsbezogenen Interpretationswissenschaft hin zu einer rechtsetzungsorientierten Handlungs- und Entscheidungswissenschaft konstatiert Voßkuhle, Methode und Pragmatik im Öffentlichen Recht, in: Bauer u.a. (Hrsg.), Umwelt, Wirtschaft und Recht, 2002, S. 171 (179ff.). Zust. Schmidt-Aßmann, Ordnungsidee (Fn. 14), 1. Kap., Rn. 46.

68 Zur Bedeutung der Rechtsvergleichung im Öffentlichen Recht aus neuerer Zeit vgl. etwa Häberle, Rechtsvergleichung im Kraftfeld des Verfassungsstaates, 1992; Krüger, Eigenart, Methode und Funktion der Rechtsvergleichung im öffentlichen Recht, in: FS für Martin Kriele, 1997, S. 1393 ff.; Starck, Rechtsvergleichung im öffentlichen Recht, JZ 1997, $1021 \mathrm{ff.;} \mathrm{Trantas,}$ Die Anwendung der Rechtsvergleichung bei der Untersuchung des öffentlichen Rechts, 1998; Sommermann, Die Bedeutung der Rechtsvergleichung für die Fortentwicklung des Staatsund Verwaltungsrechts in Europa, DÖV 1999, 1017ff.; Bullinger, Sinn und Methoden der Rechtsvergleichung im Zivilrecht und im Verwaltungsrecht, in: FS Peter Schlechtriem, 2003, S. $331 \mathrm{ff}$.

${ }^{69}$ Erinnert sei hier nur an den Einfluß des französischen Verwaltungsrechts auf die Entstehung des deutschen Verwaltungsrechts in der zweiten Hälfte des 19. Jahrhunderts, vgl. dazu Ernst v. Meier, Französische Einflüsse auf die Staats- und Rechtsentwicklung Preußens im 19. Jahrhundert, 2 Bde., 1907, 1908; Otto Mayer, Theorie des französischen Verwaltungsrechts, 1886; Heyen, Profile der deutschen und französischen Verwaltungsrechtswissenschaft 1880-1914, 1989.

${ }^{70}$ Für die Rechtsvergleichung vgl. Trantas, Rechtsvergleichung (Fn. 68), S. 27-30; Sommermann, DÖV 1999, 1017 (1028f.); Möllers, Theorie, Praxis und Interdisziplinarität in der Verwaltungsrechtswissenschaft, VerwArch 93 (2002), 22 (52-54), jeweils m. w. N. 


\section{Von der „Krise“ zur „Reform“ des Verwaltungsrechts}

Der Reformimpuls der Europäisierung und Internationalisierung sollte auf fruchtbaren Boden fallen. Spätestens seit Mitte der 1980er Jahren konstatierte man vielerorts eine Krise des Verwaltungsrechts. ${ }^{71}$ Ausgelöst worden war das Krisenszenario durch einige (wenige) viel beachtete empirische Implementationsstudien ${ }^{72}$ aus den 1970er Jahren, deren Verdienst es ist, die Aufmerksamkeit von Politik und Wissenschaft vor allem auf zwei Phänomene gelenkt zu haben: ${ }^{73}$ Zum einen zeigte sich, daß nicht unerhebliche Teile des geltenden Umweltrechts in der Praxis nicht umgesetzt wurden, mithin „Vollzugsdefizite“ bestanden. Vielleicht noch wichtiger war die zweite Erkenntnis, daß die Verwaltung in vielfältiger Weise mit den betroffenen Wirtschaftsunternehmen und Bürgern kooperierte, um gemeinsam akzeptable Lösungen für auftretende (Rechts-)Probleme zu finden. ${ }^{74}$ Offensichtlich duldeten die zuständigen Hoheitsträger nicht nur über längere Zeiträume rechtswidrige Zustände, sie entwickelten bei der Erfüllung ihrer Aufgaben auch ganz eigene Strategien und Praktiken, die den tradierten ordnungsrechtlich geprägten Verfahren und Handlungsformen zumindest auf den ersten Blick überlegen zu sein schienen. Aus diesem Grund fiel es der Verwaltungsrechtswissenschaft auch schwer, diese soziologischen Befunde unter Hinweis auf den Vorrang des Gesetzes einfach zu ignorieren, was aus dem Blickwinkel der juristischen Methode durchaus konsequent gewesen wäre, zumal die organisationserhaltende Funktion des Informalen seit längerer Zeit bekannt war. ${ }^{75}$ Statt dessen suchte man zumindest teilweise verstärkt Kontakt zu Sozial- und Politikwissenschaftlern, um mit ihnen gemeinsam die informale Zusammenarbeit zwischen Verwaltung, Wirtschaft und Bürger näher zu analysieren. ${ }^{76} \mathrm{Im}$ Vorder-

71 Pitschas, Verwaltungsverantwortung (Fn. 10), S. 48-53. Vgl. ferner Günther, Der Wandel der Staatsaufgaben und die Krise des regulativen Rechts, in: Grimm (Hrsg.), Wachsende Staatsaufgaben - sinkende Steuerungsfähigkeit des Rechts, 1990, S. 51 ff.; Wolf, Zur Antiquiertheit des Rechts in der Risikogesellschaft, Leviathan 1987, 357 ff. Krit. zur Popularität von Krisenszenarien zuletzt wieder Di Fabio, Das Recht offener Staaten, 1998, S. 9; Möllers, Theorie (Fn. 70), 26-31; Lepsius, Steuerungsdiskussion, Systemtheorie und Parlamentarismuskritik, 1999, S. 19: „Die vermeintliche Krise des Rechts als Regelungsinstrument ist keine Krise des Rechts, sondern eine Krise der gesellschaftlichen Erwartungen“.

72 Winter, Das Vollzugsdefizit im Wasserrecht, 1975; Mayntz u.a., Vollzugsprobleme der Umweltpolitik, 1978 und darauf aufbauend Bohne, Der informale Rechtsstaat, 1981. Später folgten v. Wedemeyer, Kooperation statt Vollzug im Umweltrecht, 1995 (Diss. 1991); Rüther, Die behördliche Praxis bei der Entdeckung und Definition von Umweltstrafsachen, 1991, sowie Hill/Weber, Vollzugserfahrungen mit umweltrechtlichen Zulassungsverfahren in den neuen Ländern, 1996, mit einer Zusammenfassung der Ergebnisse der bisherigen Untersuchungen, ebd., S. $21 \mathrm{ff}$. Sehr viel besser steht es um den Vollzug des Immissionsschutzrechts durch die Verwaltung nach der Untersuchung von Dose, Die verhandelnde Verwaltung, 1997, S. 62-69, 216-219, 411. Vollzugsdefizite im Gewässerschutzrecht konstatiert dagegen weiterhin Graf, Vollzugsprobleme im Gewässerschutz, 2002, S. 124-196.

${ }^{73}$ Frühe und einflußreiche verwaltungsrechtswissenschaftliche Rezeption durch HoffmannRiem, Selbstbindungen der Verwaltung, VVDStRL 40 (1981), 187 (191ff.).

${ }^{74}$ Diese Erkenntnis war zwar nicht eigentlich neu, von der Rechtswissenschaft aber bis zu diesem Zeitpunkt weitgehend ignoriert worden. Zentrale Impulse für die neuere Diskussion gingen aus von: Rittner, Der kooperative Staat, AöR 104 (1979), $389 \mathrm{ff}$.

75 So auch Möllers, Theorie (Fn. 70), $35 \mathrm{f}$.

${ }^{76}$ Die Neuaufnahme des interdisziplinären Gesprächs mit den Politik- und Sozialwissenschaftlern ist dokumentiert in verschiedenen Tagungsbänden, vgl. z. B. Hoffmann-Riem/Schmidt- 


\section{Allgemeines Verwaltungsrecht}

grund stand dabei die Typologisierung und Klassifizierung der verschiedenen Ausprägungen (paralegaler) exekutiver Handlungsweisen. ${ }^{77}$ Allerdings vermochte man innerhalb der Verwaltungsrechtswissenschaft und der Rechtsprechung aus der deskriptiv-typologischen Aufarbeitung der Verwaltungspraxis zunächst kaum produktiven (dogmatischen) Ertrag zu ziehen. Thematisiert wurden lediglich die rechtlichen Grenzen kooperativ ausgerichteter Hoheitstätigkeit, etwa die Grenzen informeller Arrangements ${ }^{78}$ oder die Grenzen des Verwaltungsvertrags. ${ }^{79}$

Erst nach und nach setzte sich die Erkenntnis durch, daß nur durch einen grundsätzlichen Umbau des Verwaltungsrechts und seiner Dogmatik dem komplexen Zusammenhang zwischen Rechtsetzung, konkreter Entscheidung und Vollzug hinreichend Rechnung getragen werden kann. Als zentrale Herausforderung entpuppt sich heute insoweit die Bewältigung der Wissensproblematik: Um ihre Aufgaben $\mathrm{zu}$ bewältigen und normative Handlungsanweisungen $\mathrm{zu}$ formulieren, benötigen Gesetzgeber und Verwaltung nicht nur entsprechende Personal- und Sachmittel, sondern auch ausreichende Informationen; erst die ausreichende Verfügbarkeit von Wissen, also solcher Informationen, die in verarbeiteter, d. h. organisierter und systematisierter Form vorliegen, schafft Handlungskapazität. ${ }^{80}$ Mit dem Wandel des bürgerlichen Rechtsstaats zum intervenierenden Wohlfahrts- und Präventionsstaat und der damit einhergehenden quantitativen Ausweitung und qualitativen Veränderung der Staatsaufgaben ${ }^{81}$ ist der Bedarf an Information und Wissen drastisch gestiegen. ${ }^{82}$ Gleichzeitig sind insbesondere in den Bereichen des

Aßmann (Hrsg.), Konfliktbewältigung durch Verhandlungen, Bd. I und II, 1990; Benz/Seidel (Hrsg.), Zwischen Kooperation und Korruption. Abweichendes Verhalten in der Verwaltung, 1992; Dose/Voigt (Hrsg.), Kooperatives Recht, 1995; Voigt (Hrsg.), Der Kooperative Staat. Krisenbewältigung durch Verhandlung?, 1995.

77 Die Diskussion zusammenfassend Dreier, Informales Verwaltungshandeln, StWStP 1993, $647 \mathrm{ff}$.

78 Vgl. z. B. Brohm, Rechtsstaatliche Vorgaben für informelles Verwaltungshandeln, DVBl. 1994, $133 \mathrm{ff}$;; Schulte, Schlichtes Verwaltungshandeln, 1995, S. $82 \mathrm{ff}$.; Tomerius, Informelle Projektabsprachen im Umweltrecht, 1995, S. $39 \mathrm{ff}$.

${ }^{79}$ Hingewiesen sei hier nur auf die Arbeiten von Spannowsky, Grenzen des Verwaltungshandelns durch Verträge und Absprachen, 1994, und Gurlit, Verwaltungsvertrag und Gesetz, 2000, S. $245 \mathrm{ff}$. Die Notwendigkeit einer "gesetzesdirigierten Vertragsgestaltung“ betont dagegen zu Recht Schmidt-Aßmann, Verwaltungsverträge im Städtebaurecht, in: FS für Konrad Gelzer, 1991, S. 117 (122 ff.). Weiterführend ferner Krebs, Verträge und Absprachen zwischen Verwaltung und Privaten, VVDStRL 52 (1993), 248 (277ff.), und Bauer, Die negative und positive Funktion des Verwaltungsvertragsrechts, in: FS für Franz Knöpfle, 1996, S. 11 (14ff.).

80 Zur Tätigkeit der Verwaltung als Informationsverarbeitung Pitschas, Allgemeines Verwaltungsrecht als Teil der öffentlichen Informationsordnung, in: Hoffmann-Riem/SchmidtAßmann/Schuppert (Hrsg.), Reform (Fn. 23), S. 227 (279ff.); Grimmer, Struktur und Innovation, 1997, S. 22-25; Voßkuhle, Der Wandel von Verwaltungsrecht und Verwaltungsprozeßrecht in der Informationsgesellschaft, in: Hoffmann-Riem/Schmidt-Aßmann (Hrsg.), Verwaltungsrecht in der Informationsgesellschaft, 2000, S. 349 (352ff.) m.w. N.

${ }^{81}$ Statt vieler vgl. Stolleis, Die Entwicklung des Interventionsstaates und das öffentliche Recht, ZNR 11 (1989), $129 \mathrm{ff}$;; Grimm, Die Zukunft der Verfassung, in: ders., Die Zukunft der Verfassung, 1991, S. 159 ff. bzw. 397 ff.; Schulze-Fielitz, Der Leviathan auf dem Weg zum nützlichen Haustier?, in: Rüdiger Voigt (Hrsg.), Abschied vom Staat - Rückkehr zum Staat?, 1993, S. $95 \mathrm{ff}$.

82 Aufschlußreich zum Wissensmanagement des Staates gerade auch in historischer Sicht die Beiträge in: Collin/Horstmann (Hrsg.), Das Wissen des Staates, 2004. 
Umwelt-, Telekommunikations- und Technikrechts die Grenzen des verfügbaren Wissens angesichts überbordender Komplexität und Dynamik, nicht-linearen Kausalverläufen, Diskontinuitäten und Irreversibilitäten, globalen Effekten sowie unabschätzbaren Risiken immer schneller erreicht. ${ }^{83}$ Das aus diesem Wissensdilemma resultierende Bedürfnis nach flexiblen, situationsbezogenen und im weitesten Sinne lernfähigen Handlungsanweisungen kann das traditionelle Ordnungsrecht nicht hinreichend befriedigen. ${ }^{84}$ Auch die klassisch bürokratisch-hierarchisch organisierte Verwaltung erscheint kaum in der Lage, einen ausreichenden Informationsfluß zu gewährleisten. Folglich sind Gesetzgeber und Verwaltungsrechtswissenschaft aufgerufen, jedenfalls in bestimmten, durch besondere Dynamik geprägten Rechtsgebieten über alternative Regelungsstrategien und Organisationsformen nachzudenken. Als wesentliche Entwicklungslinien des sich in den letzten Jahren langsam herausbildenden ,Neuen Verwaltungsrechts“ 85 lassen sich nennen:

- die Verlagerung von Verwaltungsaufgaben auf Private. Primäres Ziel ist insofern nicht nur die Entlastung des vermeintlich ,überforderten“ Staates ${ }^{86}$ durch Aufgabenabbau und Einsparung von Geld für Personal und Sachmittel, sondern vor allem eine effektivere Bewältigung öffentlicher Aufgaben durch die Mobilisierung der endogenen Potentiale der Gesellschaft, ihrer Anpassungs-, Reaktionsund Problemlösungskapazitäten. ${ }^{87}$ Statt auf klassische Take-over-Lösungen wie der Übertragung staatlichen bzw. kommunalen Eigentums auf Private (sog. Vermögensprivatisierung) oder die echte Aufgabenverlagerung in den privaten Sektor (sog. materielle Privatisierung) setzt man dabei zunehmend auf gleitende Teillösungen. $\mathrm{Zu}$ denken ist hier etwa an den Zusammenschluß öffentlicher und privater Akteure in gemischt-wirtschaftlichen Unternehmen, ${ }^{88}$ die Übertra-

83 Zur Ungewißheit als Herausforderung des (Verwaltungs)Rechts vgl. nur Scherzberg, Wissen, Nichtwissen und Ungewißheit im Recht, in: Engel/Halfmann/Schulte (Hrsg.), Wissen Nichtwissen - Unsicheres Wissen, 2002, S. $113 \mathrm{ff}$;; Appel, Methodik des Umgangs mit Ungewißheit, in: Schmidt-Aßmann/Hoffmann-Riem (Hrsg.), Methoden (Fn. 3), S. $327 \mathrm{ff}$. Zur Theorie und zum Begriff des Risikos vgl. zuletzt die gegensätzlichen Referate von Scherzberg und Lepsius, Risikosteuerung durch Verwaltungsrecht: Ermöglichung oder Begrenzung von Innovation, VVDStRL 63 (2004), 215 (219-225) bzw. 263 (266-283) m.w. N.

84 Übersichtlicher Problemaufriß bei Ritter, Von den Schwierigkeiten des Rechts mit der Ökologie, DÖV 1992, $641 \mathrm{ff}$.

85 Vgl. die übergreifenden Analysen von Bauer, Verwaltungsrechtslehre im Umbruch?, DV 25 (1992), $301 \mathrm{ff}$; Henke, Wandel der Dogmatik des öffentlichen Rechts, JZ 1992, $541 \mathrm{ff}$; Thieme, Über die Notwendigkeit einer Reform des Allgemeinen Verwaltungsrechts, DÖV 1996, 757 ff.; Hoffmann-Riem, Tendenzen der Verwaltungsrechtsentwicklung, DÖV 1997, $433 \mathrm{ff}$; Wallerath, Verwaltungserneuerung, VerwArch 88 (1997), 1 ff.; Badura, Verwaltungsrecht im Umbruch, in: Kitagawa u.a. (Hrsg.), Das Recht vor den Herausforderungen des neuen Jahrhunderts, 1998, S. $147 \mathrm{ff}$; Huber, Die entfesselte Verwaltung, StWStP 1998, $423 \mathrm{ff}$.; Schmidt, Die Reform von Verwaltung und Verwaltungsrecht, VerwArch 91 (2000), $149 \mathrm{ff}$; Voßkuhle, "Schlüsselbegriffe" der Verwaltungsrechtsreform, VerwArch 92 (2001), 184 ff.; Pitschas, Neues Verwaltungsrecht im partnerschaftlichen Rechtsstaat?, DÖV 2004, $231 \mathrm{ff.}$

86 Statt vieler Ellwein/Hesse, Der überforderte Staat, 1997.

87 Voßkuhle, Gesetzgeberische Regelungsstrategien der Verantwortungsteilung zwischen öffentlichem und privatem Sektor, in: Schuppert (Hrsg.), Jenseits von Privatisierung und schlankem Staat, 1999, S. 47 (50).

88 Allgemein dazu Gersdorf, Öffentliche Unternehmen im Spannungsfeld zwischen Demokratie- und Wirtschaftlichkeitsprinzip, 2000, S. $136 \mathrm{ff.}$ 
gung spezieller hoheitlicher Befugnisse auf Private im Rahmen der sog. Beleihung, ${ }^{89}$ die Gründung von Public Private Partnerships, die Einbeziehung privaten Kapitals bei der Finanzierung von Infrastrukturvorhaben, z. B. bei der Finanzierung von Straßen nach dem Gesetz über den Bau und die Finanzierung von Bundesfernstraßen ${ }^{90}$ (sog. Finanzierungsprivatisierung), die verschiedenen Formen sog. funktioneller Privatisierung ${ }^{91}$ mit der Unterspielart der sog. Verfahrensprivatisierung, etwa den Einsatz Privater als behördliche Verfahrensbevollmächtigte ${ }^{92}$ oder die Überwachung von Anlagen durch staatlich beauftragte Private $^{93}$ und die Etablierung unterschiedlicher Formen regulierter Selbstregulierung. ${ }^{94}$ Was freilich fehlt, ist die Bereitstellung eines Rechtsregimes, das in der Lage ist, die Einbindung Privater in die öffentliche Aufgabenwahrnehmung im Hinblick auf eine größtmögliche Gemeinwohlverwirklichung anzuleiten und abzusichern. Von daher erklären sich auch die Forderungen in der Literatur nach Entwicklung eines Privatorganisations-, Privatisierungsfolgen-, Verwaltungskooperations- oder Regulierungsverwaltungsrechts. ${ }^{95}$ Sie münden ein in die Ausbildung einer allgemeinen Dogmatik des Gewährleistungsverwaltungsrechts als dritter Säule des Verwaltungsrechts neben dem Ordnungs- und Leistungsrecht. ${ }^{96}$

- die Verdichtung der Kommunikationsbeziehungen zwischen Behörde und Bürger. ${ }^{97}$ Beispiele sind hier die gezielte Information der Öffentlichkeit durch die Verwaltung (sog. „Publikumsinformation“98) mittels Aufklärung (z.B. über die Zusammensetzung von Arzneimitteln ${ }^{99}$ ), Empfehlungen (z.B. für umweltfreund-

${ }^{89}$ Zur Wiederentdeckung der Beleihung in der Praxis vgl. Steiner, Fragen der Beleihungsdogmatik aus österreichischer und deutscher Sicht, in: FS Friedrich Koja, 1998, S. 603 ff.; Burgi, Der Beliehene - ein Klassiker im modernen Verwaltungsrecht, in: FS Hartmut Maurer 2001, S. $581 \mathrm{ff}$.

90 Gesetz über den Bau und die Finanzierung von Bundesfernstraßen durch Private vom 30. 8. 1994 (BGB1. I S. 2243), zuletzt geändert durch Gesetz vom 1. 9.2002 (BGB1. I S. 3442). Aus der Rspr. vgl. BVerwG, NVwZ 2000, 555 ff.; VfGH RPf., NVwZ-RR 1998, 145 ff. - Aus der Lit. vgl. Papst, Verfassungsrechtliche Grenzen der Privatisierung im Fernstraßenbau, 1997, S. 184 ff.; Bucher, Die Privatisierung von Bundesfernstraßen, 1996, S. $64 \mathrm{ff.}$, 176f. Vgl. ferner zu weiteren Beispielen der Finanzierungsprivatisierung Zeiss, Privatfinanzierung staatlicher Infrastruktur, 2000, und Meeder, Public Private Partnership zur Finanzierung von Flughafenprojekten in Europa, 2000.

91 Ausführlich Burgi, Funktionale Privatisierung und Verwaltungshilfe, 1999, etwa S. $145 \mathrm{ff}$.

92 Siehe $\S 2$ Abs. 2 S. 3 Nr. 5 der 9. BImSchVO und $\S 71$ c Abs. 2 S. 2 VwVfG.

93 Vgl. z. B. § 52 Abs. 2 BImSchG; 25 Abs. 3 GenTG; § 21 Abs. 4 ChemG. Näher dazu Seidel, Privater Sachverstand und staatliche Garantenstellung im Verwaltungsrecht, 2000, S. 200 ff.; Scholl, Der private Sachverständige im Verwaltungsrecht, 2005.

94 Näher dazu Schmidt-Preuß und Di Fabio, Verwaltung und Verwaltungsrecht zwischen gesellschaftlicher Selbstregulierung und staatlicher Steuerung, VVDStRL 56 (1997), 160ff. bzw. 235 ff. Vgl. auch die Beiträge in: Regulierte Selbstregulierung als Steuerungskonzept des Gewährleistungsstaats, DV Beiheft 4 (2001).

95 Eingehende Nachweise bei Voßkuhle, Beteiligung Privater an der Wahrnehmung öffentlicher Aufgaben und staatliche Verantwortung, VVDStRL 62 (2003), 266 (268f.).

96 Systematisch entfaltet bei Voßkuhle, VVDStRL 62 (2003), 266 (304ff.).

97 Näher dazu etwa Pitschas, Informationsordnung (Fn. 80), S. $227 \mathrm{ff}$; Voßkuhle, Wandel (Fn. 80), S. $365 \mathrm{ff}$.

98 Begriffsbildung und Abgrenzung bei Gramm, Aufklärung durch staatliche Publikumsinformation, Der Staat 30 (1991), 51 (53 f.).

99 Vgl. BVerwGE 71, 183 ff. (Arznei-Transparenzlisten). 
liche Produkte ${ }^{100}$ ) oder Warnungen (z. B. vor dem Genuß bestimmter Nahrungsmittel ${ }^{101}$ oder dem Eintritt in Sekten ${ }^{102}$ ), der Ausbau der Vorfeldkommunikation im Verwaltungsverfahren (z.B. durch Beratungspflichten [ $\$ 71 \mathrm{c}$ VwVfG] und die Einführung von Vor-Antragsverfahren [§ 5 UVPG; § 2 Abs. 2 9. BImSchV; $\S 71 \mathrm{c}$ Abs. 2 VwVfG]), die Erweiterung der Kommunikationsformen (z.B. durch Institutionalisierung des „,runden Tisches“ in Form der Antragskonferenz [vgl. § 71 e VwVfG]), neue Formen der informationellen Kooperation bei der Sachaufklärung (z.B. Anzeige-, Melde-, Mitteilungs-, Auskunfts- und qualifizierte Erklärungspflichten des Antragstellers) und Informationsvermittlungspflichten der Behörde (z. B. durch Informationsmittler [§ 25 Abs. 2 SGB X] oder der voraussetzungslose Anspruch auf Akteneinsicht nach dem UIG) ${ }^{103}$ Ferner ist in diesem Zusammenhang auf die neuen Möglichkeiten der Nutzung des Internet hinzuweisen (Stichwort: E-Government). ${ }^{104}$

- die Ökonomisierung des Verwaltungsrechts. ${ }^{105}$ So versucht man etwa in der Bundesrepublik - inspiriert durch Vorbilder in der amerikanischen Luftreinhaltepolitik und mit massiver Unterstützung von Politik und Wissenschaft - seit Anfang der achtziger Jahre speziell das geltende Umweltordnungsrecht unter Zuhilfenahme ökonomischer Theorievorstellungen zu reformieren. ${ }^{106}$

Maßgebliche Anstöße und Vorarbeiten zu den skizzierten Entwicklungen gehen auf den von Wolfgang Hoffmann-Riem und Eberhard Schmidt-Aßmann ins Leben gerufenen Reformkreis zurück, der auch methodisch zu einer Neuausrichtung der Verwaltungsrechtswissenschaft geführt hat. ${ }^{107}$

100 Vgl. dazu Ossenbühl, Umweltpflege durch hoheitliche Produktkennzeichnung, 1995.

101 Vgl. BVerw GE 87, 37 ff. (Glykolwein); BVerfGE 105, $252 \mathrm{ff}$.

102 Vgl. BVerwGE 82, $76 \mathrm{ff}$. (Transzendentale Meditation); 90, $112 \mathrm{ff}$; BVerfGE 105, $279 \mathrm{ff}$. (Osho-Bewegung); BVerfG, NJW 1989, 3269 ff.; BVerw G, NJW 1991, 1770 ff. (Jugendsekten).

103 BGBl. I S. 2722.

104 Vgl. statt vieler Boehme-Neßler, Electronic Government: Internet und Verwaltung, NVwZ 2001, 374 (379f.); Groß, Die Informatisierung der Verwaltung, VerwArch 95 (2004), 400 ff.; Eifert, Electronic Government, 2006. Materialreiche Überblicke ferner bei von Lucke, Regieren und Verwalten im Informationszeitalter, 2003, S. 63 ff., $119 \mathrm{ff}$; Hill, eGovernment - Mode oder Chance zur nachhaltigen Modernisierung der Verwaltung?, BayVBl. 2003, $737 \mathrm{ff}$.

${ }^{105}$ Instruktiver Überblick bei Schneider, Zur Ökonomisierung von Verwaltungsrecht und Verwaltungsrechtswissenschaft, DV 2001, $317 \mathrm{ff}$, und Fehling, Kosten-Nutzen-Analysen als Maßstab für Verwaltungsentscheidungen, VerwArch 95 (2004), 443 ff. Vgl. ferner Gröpl, Ökonomisierung von Verwaltung und Verwaltungsrecht, VerwArch 93 (2002), $459 \mathrm{ff}$.

106 Näher dazu Sparwasser/Engel/Voßkuhle, Umweltrecht, 5. Aufl. 2003, Rn. 113 ff. m. w. N.

107 Die Reformansätze sind dokumentiert in den von Hoffmann-Riem und Schmidt-Aßmann (sowie Schuppert) herausgegebenen "Schriften zur Reform des Verwaltungsrechts“, die insgesamt zehn Bände umfassen: Reform des Allgemeinen Verwaltungsrechts, 1993; Innovation und Flexibilität des Verwaltungsrechts, 1994; Öffentliches Recht und Privatrecht als wechselseitige Auffangordnungen, 1996; Verwaltungsorganisationsrecht als Steuerungsressource, 1997; Effizienz als Herausforderung an das Verwaltungsrecht, 1998; Strukturen des Europäischen Verwaltungsrechts, 1999; Verwaltungsrecht in der Informationsgesellschaft, 2000; Verwaltungskontrolle, 2001; Verwaltungsverfahren und Verwaltungsverfahrensgesetz, 2002; Methoden der Verwaltungsrechtswissenschaft, 2004. 


\section{Verwaltungsprozeßrecht}

\section{Auf- und Ausbau des verwaltungsgerichtlichen Rechtsschutzsystems in der Nachkriegszeit}

Dem seit der Revolution von 1848/49 geführten Kampf um die Einführung einer selbständigen und umfassenden Verwaltungsgerichtsbarkeit ${ }^{108}$ sollte erst unter dem Grundgesetz Erfolg beschieden sein. In der Weimarer Zeit ${ }^{109} \mathrm{kam}$ es weder zur Gründung des bereits kurz nach der Reichsgründung geplanten Reichsverwaltungsgerichts noch zum Ausbau der Verwaltungsgerichtsbarkeit in erster Instanz. Lediglich in Hamburg existierten ab 1921 ein VG und ein OVG als echte Gerichte. Ferner begrenzte das Enumerationsprinzip den Zugang zu den bestehenden Oberverwaltungsgerichten auf die Anfechtungsklage gegen belastende Verwaltungsakte. Mit dem Inkrafttreten des Grundgesetzes änderte sich die Situation grundlegend:110 Art. 19 Abs. 4 GG eröffnet bei jeder Beeinträchtigung subjektiver Rechte durch die öffentliche Gewalt den Zugang zu Gericht und garantiert damit zusammen mit Art. 92, 97 GG die Entscheidung von Verwaltungsstreitsachen durch organisatorisch unabhängige staatliche Gerichte mit sachlich und persönlich unabhängigen Richtern. ${ }^{111}$ Gleichzeitig ist dort ein umfassender Anspruch auf effektiven Rechtsschutz gewährleistet. Bereits vor Erlaß des Grundgesetzes hatte man in der amerikanischen und britischen Zone unter Mitwirkung von Vertretern aus Gerichtspraxis und Wissenschaft im beratenden Ausschuß ${ }^{112}$ mit der Errichtung von Verwaltungsgerichten begonnen. Die Verwaltungsgerichtsgesetze der süddeutschen Länder ${ }^{113}$ und die in der britischen Zone geltende Militärgerichtsverordnung Nr. 165 vom 15. September $1948^{114}$ können als Vorläufer einer einheitlichen Prozeßkodifikation angesehen werden. Ihre Kommentierung durch die Habilitationsschriften von Bachof ${ }^{115}$ und Menger ${ }^{116}$ sowie eine Reihe grundlegender Aufsätze ${ }^{117}$ bildeten im folgenden Jahrzehnt die Basis für die Entwicklung des Verwaltungsprozeßrechts als eigenständige dogmatische Disziplin. ${ }^{118}$ Seiner Verpflichtung

108 Übersichtlich: Sydow, Die Revolution von 1848/49: Ursprung der modernen Verwaltungsgerichtsbarkeit, VerwArch 92 (2001), $317 \mathrm{ff}$.

${ }_{109} \mathrm{Zu}$ ihr im vorliegenden Zusammenhang etwa Grawert, Verwaltungsrechtsschutz in der Weimarer Republik, in: FS für Menger, 1985, S. $35 \mathrm{ff}$.

110 Zum folgenden vgl. z.B. Ule, Die geschichtliche Entwicklung des verwaltungsgerichtlichen Rechtsschutzes in der Nachkriegszeit, in: FS für Menger, 1985, S. 81 ff.; von Unruh, Verwaltungsgerichtsbarkeit, in: Jeserich/Pohl/von Unruh, Verwaltungsgeschichte (Fn. 3), S. $1178 \mathrm{ff}$; Papier, Die Stellung der Verwaltungsgerichtsbarkeit im demokratischen Rechtsstaat, 1979, S. 7, $13 \mathrm{ff} ., 19 \mathrm{ff}$.

111 So statt vieler Schulze-Fielitz, in: Dreier (Hrsg.), Grundgesetzkommentar, 2. Aufl. 2004, Art. 19 Abs. 4, Rn. 90 m.w. N.

112 Vgl. Jellinek, DRZ 1948, 470 ff.; Ule, Entwicklung (Fn. 110), S. 83 f.

113 Nachweise bei Schmidt-Aßmann, in: Schoch/Schmidt-Aßmann/Pietzner (Hrsg.), VwGO, Einleitung, Rn. 83.

114 VOBl BZ 1948, S. 263.

115 Bachof, Die verwaltungsgerichtliche Klage auf Vornahme einer Amtshandlung, 1951.

116 Menger, System des verwaltungsgerichtlichen Rechtsschutzes, 1954.

$117 \mathrm{Vgl}$. etwa die repräsentativen Beiträge in der GS für Jellinek, 1955.

118 So Schmidt-Aßmann, Einleitung (Fn. 113), Rn. 90. 
aus Art. 95 Abs. 1 GG, ein Bundesverwaltungsgericht zu errichten, ist der Bundesgesetzgeber im Jahre 1953 nachgekommen. ${ }^{119}$ Bis zum Inkrafttreten der Verwaltungsgerichtsordnung am 21. Januar 1960, ${ }^{120}$ die das Recht der Verwaltungsgerichtsbarkeit und das verwaltungsgerichtliche Vorverfahren auf eine einheitliche Grundlage stellte, sollten aber noch sieben Jahre vergehen. Der in der Nachkriegszeit begonnene Prozeß der Vereinheitlichung fand damit seinen vorläufigen Abschluß; die prägenden Elemente des verwaltungsgerichtlichen Rechtsschutzsystems waren nun in einem Gesetz zusammengefaßt: ${ }^{121}$ dreistufiger Gerichtsaufbau ( $\$ 2$ VwGO), Überwindung des Enumerationsprinzips durch Einführung der verwaltungsprozessualen Generalklausel ( $\$ 40 \mathrm{VwGO}$ ), subjektiv-rechtliche Ausrichtung der Klagearten ( $\S 42$ Abs. 2, 113 Abs. 1 S. 1, Abs. 5 VwGO), eingeschränkte gerichtliche Kontrolle verwaltungsbehördlicher Ermessensentscheidungen ( 114 VwGO).

\section{Rechtsschutzhypertrophie: Die Ausweitung der Klagebefugnis und der gerichtlichen Kontrolldichte}

In der Folgezeit ${ }^{122}$ galt das Bemühen der Rechtsprechung und Literatur vor allem der rechtsschutzfreundlichen Auslegung und Handhabung der neuen Verwaltungsprozeßordnung. Als zentraler Interpretationshebel diente dabei Art. 19 Abs. 4 GG und das dort verankerte Gebot eines effektiven Rechtsschutzes. ${ }^{123}$ Ausprägungen des Wirksamkeitsgebots sind u.a. das Gebot der Rechtswegklarheit, Vorwirkungen auf die Ausgestaltung des Verwaltungsverfahrens, die Institute des vorbeugenden Rechtsschutzes und des Rechtsschutzes gegen erledigtes Verwaltungshandeln, die Rechtssicherung vor und während des gerichtlichen Verfahrens durch die Institute des vorläufigen Rechtsschutzes, der Grundsatz vollständiger gerichtlicher Rechtskontrolle, demzufolge eine Letztentscheidungsbefugnis der Verwaltung nur in Ausnahmefällen anerkannt ist (sog. normative Ermächtigungslehre) und das Gebot zeitgerechter Entscheidung. ${ }^{124}$ Daneben spielte die Ausdehnung des subjektiven öffentlichen Rechts durch die Entwicklung der Schutznormlehre eine wesentliche Rolle für die Ausweitung der Rechtsschutzmöglichkeiten des Bürgers, der nun nicht mehr Adressat einer Verwaltungsmaßnahme sein mußte, um gegen dieselbe eine zulässige Klage erheben zu können. ${ }^{125}$

119 Gesetz über das Bundesverwaltungsgericht vom 23. 9. 1952 (BGB1. I, S. 625). Durch Führererlaß war bereits am 3. 4. 1941 das Reichsverwaltungsgericht aus der Taufe gehoben worden; seine Errichtung war aber aufgrund der nicht gewährleisteten Unabhängigkeit der Richter und der Mitwirkung von NSDAP-Funktionären als ehrenamtliche Richter eine „Farce“, so zutreffend Hufen, Verwaltungsprozessrecht, 6. Aufl. 2005, Rn. 19. Ausführlich Kohl, Das Reichsverwaltungsgericht, 1991.

120 BGBl. I S. 17 und 44.

121 Grundlegend Menger, System (Fn. 116).

122 Zur Entwicklungsgeschichte der Verwaltungsgerichtsbarkeit vgl. etwa Franßen, 50 Jahre Verwaltungsgerichtsbarkeit in der Bundesrepublik Deutschland, DVBl. 1998, $413 \mathrm{ff}$.

123 Vgl. BVerfGE 24, 33 (48ff.); 35, 382 (401 ff.); 61, 82 (109ff.); 96, 27 (39ff.); 100, 313 (364f.); 103, 142 (156ff.); BVerfG, NJW 2003, $1924 \mathrm{ff.}$

124 Vgl. statt vieler Schmidt-Aßmann, in: Maunz/Dürig, GG, Art. 19 Abs. 4, Rn. $229 \mathrm{ff}$. m. w. N.

125 Eingehend dazu Huber, Konkurrenzschutz im Verwaltungsrecht, 1991; Schmidt-Preuß, Kollidierende Privatinteressen im Verwaltungsrecht, 1992; Schoch, Vorläufiger Rechtsschutz und 
Der nicht geringe Preis für diese Großzügigkeit der Rechtsschutzgewährung waren die bis Ende der 1990er Jahre stetig steigenden Eingangszahlen bei den Verwaltungsgerichten, die sich angesichts begrenzter Personal- und Sachmittelressourcen in einer oft überlangen Dauer von Gerichtsverfahren ${ }^{126}$ und der verfassungsrechtlich bedenklichen Verlagerung der rechtlichen Konfliktlösung in den Bereich des vorläufigen Rechtsschutzes niederschlugen. Besonders anschauliche Beispiele für eine gewisse Hypertrophisierung des deutschen Verwaltungsrechtsschutzes sind Massenverfahren mit tausenden von Klägern im Zusammenhang mit der Realisierung größerer Infrastrukturprojekte (Flughäfen, Eisenbahntrassen, Autobahnen usw. $)^{127}$ oder die Überflutung der Verwaltungsgerichte mit asyl- und ausländerrechtlichen Verfahren.

\section{Entlastungs- und Beschleunigungsgesetzgebung}

Dem steten Ruf nach Entlastung der Verwaltungsgerichtsbarkeit sowie nach Beschleunigung und Vereinfachung des Verfahrens hat der Gesetzgeber Taten folgen lassen. Hervorzuheben sind hier das Gesetz zur Entlastung der Gerichte in der Verwaltungs- und Finanzgerichtsbarkeit vom 31. März 1978, ${ }^{128}$ das Gesetz zur Neuregelung des verwaltungsgerichtlichen Verfahrens (4. VwGOÄndG) vom 17. Dezember 1990, ${ }^{129}$ das Gesetz zur Entlastung der Rechtspflege vom 11. Januar 1993, ${ }^{130}$ das Gesetz zur Beschleunigung von Genehmigungsverfahren vom 12. September 1996, ${ }^{131}$ das 6. Gesetz zur Änderung der Verwaltungsgerichtsordnung und anderer Gesetze vom 1. November 1996. ${ }^{132} \mathrm{Zu}$ den dort vorgesehenen Maßnahmen gehören u.a. das Institut des Gerichtsbescheids, reduzierte Begründungspflichten für bestimmte gerichtliche Entscheidungen, die Einführung von Präklusionsvorschriften, die fingierte Klagerücknahme bei Nichtbetreiben des Prozesses ( $\$ 92$ Abs. 2, 126 Abs. 2 VwGO), die grundsätzliche Einführung der Zulassungsberufung und der Zulassungsbeschwerde ( $\$ 124,146$ VwGO) und die Verschärfung der Antragsbefugnis bei der Normenkontrolle ( 47 Abs. 2 VwGO). Dieses eindrucksvolle Arsenal wird zusätzlich ergänzt durch die mit dem Gesetz zur Beschleunigung von Genehmigungsverfahren vom 12. September $1996^{133}$ umgesetzte Abwertung des Verwaltungsverfahrensrechts ${ }^{134}$ sowie durch einschneidende Einschränkungen des Rechtsschutzes im Asylverfahrensgesetz und das Entfallen der aufschiebenden Wirkung baurechtlicher Nachbarwidersprüche und -klagen

Risikoverteilung im Verwaltungsrecht, 1992, insbes. S. $933 \mathrm{ff}, 1003 \mathrm{ff}$; Frenz, Verwaltungsgerichtlicher Rechtsschutz in Konkurrenzsituationen, 1999.

126 Vgl. dazu Ule, Rechtstatsachen zur Dauer des Verwaltungs-(Finanz-)Prozesses, 1977; Rottleuthner/Rottleuthner-Lutter, Die Dauer von Gerichtsverfahren, 1990, bes. S. 47-100.

127 Gegen die Planung des Großflughafens München II suchten 5724 Kläger um Rechtsschutz nach, s. Hufen, Verwaltungsprozessrecht (Fn. 119), § 3, Rn. 4.

128 BGBl. I S. 445.

129 BGBl. I S. 2809.

130 BGBl. I S. 1626.

131 BGBl. I S. 1354.

132 BGBl. I S. 1626.

133 BGBl. I S. 1354.

134 Vgl. oben B I. 3. 
( $\$ 212 \mathrm{a}$ BauGB). Lediglich die beiden zuletzt genannten Maßnahmen(bündel) und die in der Zwischenzeit durch das Gesetz zur Bereinigung des Rechtsmittelrechts im Verwaltungsprozeß vom 20. Dezember $2001^{135}$ wieder abgeschaffte Einführung der Zulassungsberufung haben in der Praxis tatsächlich Wirkung gezeigt. Im übrigen hat die Rechtsprechung unter Beifall der ganz überwiegenden Literatur $^{136}$ nicht wenigen Neuerungen im Wege der verfassungskonformen Auslegung der jeweiligen Vorschrift die Schärfe genommen. ${ }^{137}$

\section{Europäisierung des Verwaltungsprozesses}

Noch nicht absehbar sind die Konsequenzen der europäischen Einigung für die Verwaltungsgerichtsbarkeit. Parallel zur Europäisierung des Verwaltungsrechts ${ }^{138}$ wirkt das Gemeinschaftsrecht auch zunehmend in vielfältiger und eindringlicher Weise auf das nationale Verwaltungsprozeßrecht ein. ${ }^{139}$ Folgende Beispiele mögen hiervon einen Eindruck vermitteln: die Vorlagepflichten deutscher Gerichte an den EuGH bei Zweifeln über die Auslegung von Gemeinschaftsrecht (Art. 234 EG), die Erweiterung der Klagebefugnis bei möglicher Verletzung des Gemeinschaftsrechts, ${ }^{140}$ Relativierungen des vorläufigen Rechtsschutzes zur Gewährleistung der wirksamen Durchsetzung des Gemeinschaftsrechts ${ }^{141}$ oder die Erweiterung der Rechtsschutzmöglichkeiten im öffentlichen Vergabewesen. ${ }^{142}$ Problemfelder tun sich ferner etwa auf bei der Kompetenzabgrenzung zwischen der europäischen und der nationalen Gerichtsbarkeit ${ }^{143}$ oder der Anwendung des Grundsatzes der gemeinschaftskonformen Auslegung. Die Rechtswissenschaft hat auf diesen Ent-

135 BGB1. I S. 3987.

136 Zur Kritik an den Reformmaßnahmen vgl. etwa Kopp, Änderungen der Verwaltungsgerichtsordnung zum 1. Januar 1991, NJW 1991, 521 ff.; Redeker, Neue Experimente mit der VwGO?, NVwZ 1996, $521 \mathrm{ff}$; Schenke, „Reform“ ohne Ende - Das Sechste Gesetz zur Änderung der Verwaltungsgerichtsordnung und anderer Gesetze (6. VwGOÄndG), NJW 1997, $81 \mathrm{ff}$; Meissner, Die Novellierung des Verwaltungsprozeßrechts durch das Sechste Gesetz zur Änderung der Verwaltungsgerichtsordnung, VBlBW 1997, $81 \mathrm{ff}$; Stelkens, Aktuelle Probleme und Reformen in der Verwaltungsgerichtsbarkeit, NVwZ 2000, $155 \mathrm{ff}$. Vgl. auch die N. oben in Fn. 40.

137 So zutreffend Hufen, Verwaltungsprozessrecht (Fn. 119), § 3, Rn. 6.

138 Vgl. B I. 4.

139 Zum folgenden etwa Burgi, Deutsche Verwaltungsgerichte als Gemeinschaftsgerichte, DVB1. 1995, 772ff.; Classen, Die Europäisiserung der Verwaltungsgerichtsbarkeit, 1996; Stern, Die Einwirkungen des europäischen Gemeinschaftsrechts auf die Verwaltungsgerichtsbarkeit, JuS 1998, 768 ff.; Kokott, Europäisierung des Verwaltungsprozeßrechts, DV 31 (1998), 335 ff.; Schoch, Die Europäisierung des verwaltungsgerichtlichen Rechtsschutzes, 2000; Sommermann, Konvergenzen im Verwaltungsverfahrens- und Verwaltungsprozeßrecht europäischer Staaten, DÖV 2002, 133 ff.; Götz, Europarechtliche Vorgaben für das Verwaltungsprozeßrecht, DVBl. 2002, $1 \mathrm{ff}$.

140 Die Entwicklung ist hier noch im Fluß, vgl. den Überblick bei Wahl, in: Schoch/ Schmidt-Aßmann/Pietzner (Hrsg.), VwGO, Vorb. § 42 Abs. 2, Rn. 121 ff. m. w. N.

${ }^{141} \mathrm{EuGH}, \mathrm{NJW}$ 1997, 1225 (T. Port - Bananenmarktordnung); NJW 1996, 1333ff. (Atlanta); EuGH, EuZW 1990, $384 \mathrm{ff}$.

${ }^{142} \mathrm{Zu}$ den Einflüssen des gemeinschaftsrechtlichen Sekundärrechts vgl. eingehend statt vieler Drügemöller, Vergaberecht und Rechtsschutz, 1999.

143 BVerfGE 37, $271 \mathrm{ff}$. (Solange I); 73, 339 (Solange II); 89, 155 (Maastricht); 102, $147 \mathrm{ff}$. (Bananenmarkt-VO). 
wicklungsprozeß bisher erstaunlich wenig Einfluß nehmen können; ihre Aufgabe besteht eher in der Aufbereitung und Vermittlung der gemeinschaftsrechtlichen Einflüsse für die Praxis.

\section{Gegenwartsprobleme und Funktionswandel der Verwaltungsgerichtsbarkeit}

Unabhängig von der Europäisierung führen auch die oben skizzierten Veränderungen im Bereich des Verwaltungsrechts $\mathrm{zu}$ einer Aufgabenerweiterung und einem Funktionswandel innerhalb der Verwaltungsgerichtsbarkeit. ${ }^{144}$ An dieser Stelle soll nur auf drei Problemfelder hingewiesen werden:

- Sehr viele rechtlich zu beurteilende Sachverhalte sind heute geprägt durch mehrpolige Interessenstrukturen, wie sie herkömmlicherweise die Nachbarund Konkurrentenschutzklage kennzeichnen. Entsprechende Konstellationen finden sich nicht nur im Planungs- und Beamtenrecht, sondern auch in weiten Teilen des Vergabe-, Medien-, Umwelt- und Informationsrechts. Da die dahinterstehenden Konfliktlagen und komplexen Entscheidungssituationen vom Gesetzgeber kaum hinreichend verläßlich antizipiert und vorstrukturiert werden können, obliegt es hier den Verwaltungsgerichten zusammen mit der Verwaltung im Wege ,pluralisierender Rechtserzeugung“ (Brohm) ein praktikables Kollisions- und Distributionsrecht zu schaffen. ${ }^{145}$

- Vor erheblichen kognitiven Schwierigkeiten stehen die Verwaltungsgerichte ferner bei der immer häufiger an sie herangetragenen Beurteilung komplexer technischer, wirtschaftlicher und sozialer Sachverhalte, etwa wenn es darum geht, Risikoprognosen im Gentechnikrecht zu kontrollieren ${ }^{146}$ oder die Verfolgungsgefahr in fernen Bürgerkriegsgebieten im Rahmen asylrechtlicher Entscheidungen $^{147}$ abzuschätzen. Sie müssen hier den richtigen Weg finden zwischen der Skylla einer Auslieferung an die Vorbewertungen der Exekutive und der Charybdis einer Abhängigkeit von externen Sachverständigen.

- Noch schwieriger gestalten sich die Rechtsschutzprobleme in solchen Bereichen, in denen Staat und Gesellschaft, Verwaltung und Wirtschaft bei der Wahrnehmung öffentlicher Aufgaben im Wege der Verantwortungsteilung zusammenwirken. ${ }^{148}$ Oft ist hier schon nicht klar, ob sich eine Handlung überhaupt als Ausübung hoheitlicher Gewalt qualifizieren läßt und welches Rechts-

\footnotetext{
144 Zum folgenden vgl. insbes. Schmidt-Aßmann, Aufgaben- und Funktionswandel der Verwaltungsgerichtsbarkeit vor dem Hintergrund der Verwaltungsrechtsentwicklung, VBlBW 2000, 45 ff.; ders., in: Maunz/Dürig, GG, Art. 19 Abs. 4, Rn. 2 ff.; Schoch, Die Verwaltungsgerichtsbarkeit vor den Herausforderungen der jüngeren Rechtsentwicklung - Einführung in die Thematik, VBlBW 2000, $41 \mathrm{ff}$, jeweils m. w. N.

145 Vgl. statt vieler Brohm, NVwZ 1988, $794 \mathrm{ff.}$; Schmidt-Aßmann, Einleitung (Fn. 113), Rn. 176.

146 Pointiert Kirchberg, Verwaltungsgerichtsbarkeit heute - illustriert anhand der Rechtsprechung zum Elektrosmog. Eine Streitschrift, NVwZ 1998, 441 ff. Vgl. ferner Wahl, Risikobewertung der Exekutive und richterliche Kontrolldichte, NVwZ 1991, $410 \mathrm{ff}$.; Hendler, Umweltrechtliche Grenzwerte in der Gerichts- und Verwaltungspraxis, DÖV 1998, $481 \mathrm{ff}$.

147 Vgl. Groß/Kainer, Die Verteilung der Verantwortung für die Tatsachenermittlung im Asylrecht, DVB1. 1997, $1315 \mathrm{ff}$.

148 Eindringlich Schmidt-Aßmann, in: Maunz/Dürig, GG, Art. 19 Abs. 4, Rn. 3.
} 
regime zur Anwendung kommt. Teilweise wandern Konflikte sogar ganz in das Privatrecht ab, etwa im neuen Vergaberecht.

Bei der Entwicklung konzeptioneller Lösungsansätze werden die Gerichte ebenso wie der Gesetzgeber ohne Vorarbeiten der Rechtswissenschaft kaum erfolgreich sein können. Das sagt über den tatsächlichen Einfluß juristischer Standardwerke aber noch wenig aus.

\section{Verarbeitung und Beeinflussung der Rechtsentwicklungen durch juristische Standardwerke}

\section{Lehrbücher}

\section{Fundamente}

a) Fortführung und Weiterentwicklung des Erbes von Otto Mayer

Nach dem Ende des Zweiten Weltkriegs und der Herrschaft der Nationalsozialisten knüpfte man in der verwaltungsrechtlichen Praxis wie auch in der sich erst langsam wieder neu formierenden Verwaltungsrechtswissenschaft an den erreichten Stand von 1930 an. ${ }^{149}$ In kaum einem Werk der Zeit kommt diese Rückkehr zum klassischen Verwaltungsrecht unter veränderten Vorzeichen auch äußerlich so gut zum Ausdruck wie in dem „Lehrbuch des Verwaltungsrechts“ von Walter Jellinek, dessen 3. Auflage aus dem Jahre 1931 im Jahre 1948 einen Neudruck erlebte, der 1950 durch einen Nachtrag des Autors selbst ergänzt wurde. Von vielen als „didaktische Meisterleistung“" 150 und als bedeutendste Frucht der Verwaltungsrechtslehre der Weimarer Zeit eingeschätzt, stand das Lehrbuch von Jellinek ganz in der Tradition des liberalen rechtsstaatlichen Verwaltungsrechts, wie sie von Otto Mayer, seinem Lehrer, zum Ausgang des 19. Jahrhunderts begründet worden war. ${ }^{151}$ Ausgehend von der juristischen Methode und unter ,durchgängiger Reduktion auf die Rechtsform" "152 entfaltet Jellinek zunächst in einem Allgemeinen Teil systematisch alle wesentlichen Institute und Grundprinzipien des Verwaltungsrechts, bevor in einem Besonderen Teil die Mittel, ,durch die der verwaltende Staat befähigt wird, seinen Aufgaben gerecht zu werden“, 153 eingehend dargestellt werden, z.B. die Menschen ( $\$ 16$ Öffentlicher Dienst), das Geld ( 17 Grundzüge des Finanzrechts), bestimmte Sachen ( $\$ 18$ Enteignung und öffentlichrechtliche Eigentumsbeschränkung, § 22 Öffentliche Sachen und Anstalten) und Dienstleistungen (§ 19 Öffentliche Lasten). Der Besondere Teil dient gleichzeitig dazu, die Grundstrukturen praktisch wichtiger Einzelgebiete des Verwaltungsrechts heraus-

149 Deutlich Stolleis, Verwaltungsrechtswissenschaft (Fn. 3), S. 232-236. Ein Überblick über die erste Phase der Lehrbuchliteratur findet sich bei Bachof, Das Verwaltungsrecht im Spiegel der Rechtslehre, JZ 1951, $538 \mathrm{ff}$.

150 Faber, Verwaltungsrecht, 4. Aufl. 1995, S. 10.

151 Otto Mayer (Fn. 2).

152 Stolleis, Geschichte des Öffentlichen Rechts, Bd. III, 1999, S. 239.

153 Jellinek, Lehrbuch des Verwaltungsrechts, Neudruck der 3. Aufl. 1966, S. 115. 
zuarbeiten, namentlich des Beamtenrechts, des Polizeirechts, des Kommunalrechts und des Schulrechts. Gerade in dieser Einbeziehung des positiven Rechts und der Rechtsprechung besteht die wesentliche Neuerung gegenüber dem stark abstrahierenden „Verwaltungsrecht“ von Otto Mayer. Gleichzeitig ergänzte Jellinek das von Mayer herausgearbeitete Arsenal an Rechtsfiguren und Instituten um zentrale neue Kategorien wie z.B. die der ,schlichten Hoheitsverwaltung “"154 - eine Kategorie, die Geschichte gemacht hat. Aufgrund der aufbereiteten Materialfuille und zahlreicher lebensnaher Beispiele stellte sein Lehrbuch auch in der jungen Bundesrepublik eine Fundgrube für den praktisch arbeitenden Verwaltungsjuristen dar. ${ }^{155}$

Die Rechtsentwicklung unter dem Grundgesetz konnte Jellinek entgegen seinem ursprünglichen Plan von einer vierten Auflage aber nicht mehr berücksichtigen. Dies sollte der 1956 begonnenen dreibändigen Darstellung des Verwaltungsrechts von Hans Julius Wolff vorbehalten bleiben, die zunächst von Otto Bachof fortgeführt wurde (Band I ab der 9. Aufl. 1974; Band II ab der 4. Aufl. 1976; Band III ab der 4. Aufl. 1978). Nach einer zeitlichen Zäsur von 20 Jahren haben nun Rolf Stober (ab der 10. Aufl. des 1. Bandes, 1994), Winfried Kluth (ab der 6. Aufl. des 2. Bandes, 2000 und der 5. Aufl. des 3. Bandes, 2004) und Andreas Peilert (ab der 6. Aufl. des 2. Bandes, 2000) die Neubearbeitung übernommen. Obwohl in der Beck'schen Kurz-Lehrbuch-Reihe erschienen, handelte es sich bei diesem Standardwerk von Anfang an mehr um ein enzyklopädisch angelegtes „Kurzhandbuch“, ${ }^{156}$ das schon aufgrund seiner extremen Untergliederung, dem steten Wechsel von Normaldruck und Kleindruck und der minutiösen Verarbeitung der Gesetzeslage in Bund und Ländern sowie von Rechtsprechung und Literatur im Text zur durchgehenden Lektüre nicht ermunterte. ${ }^{157}$ Wer aber fortan zielgerichtet Auskunft über ein Detail der aktuellen Verwaltungsrechtsdogmatik suchte, der fand sie zuverlässig und prägnant im ,Wolff/Bachof“, dessen Verdienst es ist, die gewaltig anwachsende Stoffmenge des Verwaltungsrechts in den ersten drei Jahrzehnten der Bundesrepublik systematisch gebändigt und durch große terminologische Prägnanz handhabbar gemacht zu haben. Weitreichende Impulse für eine Fortentwicklung des Verwaltungsrechts waren insofern schon vom Ansatz her nicht intendiert ${ }^{158}$ und entsprachen auch nicht dem methodischen Zugang der

${ }^{154}$ Jellinek, Lehrbuch (Fn. 153), S. 21-24. Eine wichtige Neuerung ist ferner der ,zweiseitige Verwaltungsakt“" (a.a.O., S. 249-254).

155 Hervorgehoben von Bachof in seinem Vorwort zum Neudruck von 1966 (Fn. 153), S. IX.

156 So die Selbstcharakterisierung im Vorwort der 9. Aufl. von Band I im Jahre 1974. Deutlich auch Kellner, Besprechung, NJW 1974, 1549 (1590): „,mehr Nachschlagewerk als Lesebuch".

157 Einsichtig bereits Wolff, Verwaltungsrecht I, 1956, S. 5: „,Es wird deshalb, zumal für den Anfänger, nicht leicht eingängig zu lesen sein, sondern muß geistig aufmerksam erarbeitet werden was auch ein heilsamer Zwang ist." Zustimmend etwa Ule in einer Besprechung der 1. Aufl. von Band I (VerwArch 48 [1957], S. 86 [87]): ,Gelegentlich muß man sich fragen, ob die Neigung des Verfassers zu begrifflichen Unterscheidungen nicht dazu geführt hat, mit diesen Unterscheidungen zu weit zu gehen, zumal in der Zusammenfassung in klare und scharfe Begriffe eine gewisse didaktische Schwäche liegt ... .“ Noch deutlicher Hans Peter Ipsen, AöR 82 (1957), S. 132 (136): „Das Buch ist in diesem Sinne überhaupt nur in wenigen Partien ,lesbar"...".

${ }^{158} \mathrm{Zu}$ eigenen Standpunkten vgl. aber die eingehende Besprechung von Ule (Fn. 157). 
Autoren, denen zufolge es die Aufgabe der dogmatischen Verwaltungsrechtswissenschaft ist, ,das auf Grund der Verfassung, des Gewohnheitsrechts, der formellen Gesetze, der Rechtsverordnungen und der Praxis der Verwaltungsbehörden und Gerichte positiv geltende Verwaltungsrecht interpretierend“ darzustellen. ${ }^{159}$

\section{b) Die Öffnung des Verwaltungsrechts für die Verwaltungswirklichkeit}

Für eine Fortentwicklung des Verwaltungsrechts unter Berücksichtigung der veränderten Verwaltungswirklichkeit des modernen Sozialstaats setzten sich dagegen in den ersten Jahren der Bundesrepublik Hans Peters und vor allem Ernst Forsthoff ${ }^{160}$ ein.

Peters ging es in seinem „Lehrbuch der Verwaltung“ um eine Verschmelzung der Verwaltungsrechts- mit der Verwaltungswissenschaft. ${ }^{161}$ Neben den bestehenden gesetzlichen Regelungen versuchte er auch die außerrechtlichen Bestimmungsfaktoren des Verwaltungshandelns zu erfassen, was sich z.B. an den behandelten Fragen der Personalführung gut ablesen läßt. ${ }^{162}$ Dahinter stand die Erkenntnis, daß die Verwaltung ihr Handeln nicht nur am Kriterium der Rechtmäßigkeit, sondern darüber hinaus auch an dem der Entscheidungsrichtigkeit orientieren soll. ${ }^{163}$ Während dieses Anliegen seit den 1990er Jahren wieder stärker Beachtung findet, ${ }^{164}$ fiel es damals kaum auf fruchtbaren Boden, da Wissenschaft und Praxis offensichtlich zu stark mit der Verarbeitung der normativen Einflüsse des Grundgesetzes beschäftigt waren. ${ }^{165}$

Auch Forsthoff, ,zweifellos der bedeutendste Kopf der damaligen Verwaltungsrechtslehre", ${ }^{166}$ plädierte für eine stärkere Berücksichtigung der Verwaltungswirklichkeit. ${ }^{167}$ In seinem zur Hälfte bereits während des Krieges geschriebenen Lehrbuch, ${ }^{168}$ das 1950 im Beck-Verlag erschien und über fast drei Jahrzehnte die erste Autorität im Verwaltungsrecht darstellte (10. Auflage 1974), heißt es: „Noch hat die Verwaltungsrechtswissenschaft die Aufgabe kaum erkannt, die ihr ... gestellt

159 Wolff/Bachof, Verwaltungsrecht I, 9. Aufl. 1974, S. 58. Siehe ferner Wolff, Verwaltungsrecht I, 1956, S. 5: „Eindeutigkeit und Systematik sind denn auch das Hauptanliegen dieses Werkes, dem es mithin weit weniger auf Erörterung als auf Bestimmung ankommt“.

160 Forsthoff, Lehrbuch des Verwaltungsrechts, Band I, Allgemeiner Teil, 1. Aufl. 1950.

161 Peters, Lehrbuch der Verwaltung, 1949, S. III ff., $14 \mathrm{ff}$.

162 Peters, Lehrbuch (Fn. 161), S. $22 \mathrm{ff} ., 119 \mathrm{ff} ., 171 \mathrm{ff} ., 232 \mathrm{ff}$. Näher dazu Grigoleit/Kersten, Hans Peters (1896-1966), DV 30 (1997), 365 (388ff.).

163 Peters, Lehrbuch (Fn. 161), S. $11 \mathrm{ff}$. Auf diesen Aspekt und Parallelen zum Werk von Hoffmann-Riem hat Bumke, Methodik (Fn. 3), S. 91 hingewiesen.

$164 \mathrm{Vgl}$. insbes. Hoffmann-Riem, Verwaltungsrechtsreform. Ansätze am Beispiel des Umweltschutzes, in: ders./Schmidt-Aßmann/Schuppert (Hrsg.), Reform (Fn. 23), S. 115 (130 ff.). Ihm folgend Schmidt-Aßmann, Ordnungsidee (Fn. 14), 6. Kap., Rn. 57 m. w. N.

165 Vgl. oben B I. 1.

166 Stolleis, Verwaltungsrechtswissenschaft (Fn. 3), S. 235.

167 Näher zu seinem methodischen Ansatz Storost, Die Verwaltungsrechtslehre Ernst Forsthoffs als Ausdruck eines politischen Verfassungsmodells, in: Heyen (Hrsg.), Wissenschaft und Recht der Verwaltung seit dem Ancien Régime, 1984, S. 163 ff.; Paust, Die institutionelle Methode im Verwaltungsrecht, 1997, S. $41 \mathrm{ff}$; Schütte, Progressive Verwaltungsrechtswissenschaft auf konservativer Grundlage. Zur Verwaltungsrechtslehre Ernst Forsthoffs, 2006.

168 Zur Entstehungsgeschichte Doehring, Ernst Forsthoff, in: Juristen im Portrait, FS für den C. H. Beck Verlag, 1988, S. 346 f. 
ist: eine von Grund auf veränderte Verwaltungswirklichkeit mit dem Rechtsstaat zu verbinden oder besser: den Rechtsstaat von der heutigen Wirklichkeit aus neu zu denken, um auf diese Weise in neuen Formen das höchste Maß an rechtlichen Garantien für die Gegenwart zu retten." ${ }^{169}$ Im Mittelpunkt seiner Analyse stehen dabei die neuen Aufgaben der Verwaltung als „Leistungsträger“, die im zentralen Begriff der ,Daseinsvorsorge“ zusammengefaßt werden. ${ }^{170}$ Der dogmatische Ertrag seiner Überlegungen bleibt aber erstaunlich gering. ${ }^{171}$ Vielmehr orientiert sich Forsthoff bei der Ordnung des Rechtsstoffes im wesentlichen weiter an jenen Kategorien, Instituten und Figuren, die bereits Otto Mayer eingeführt und verwandt hat, ohne sie fortzuentwickeln. ${ }^{172}$ Wahrscheinlich stieß das Werk auch deshalb auf so große Akzeptanz: ${ }^{173}$ Es nährte die Illusion, mit der tradierten Dogmatik die Veränderungen der Verwaltungswirklichkeit in den Griff zu bekommen.

\section{c) Systematisierung von Einzelbereichen}

Neben der Gesamtdarstellung des Verwaltungsrechts darf auch die Systematisierungsarbeit in Einzelbereichen nicht unerwähnt bleiben. Ein schönes Beispiel bietet hier das Lehrbuch zum „Staatshaftungsrecht“ von Fritz Ossenbühl, das zunächst als Studienbuch in der Beck'schen JuS-Schriftenreihe erschien (1976) und heute in fünfter Auflage (1998) als großes Lehrbuch der ,grünen Reihe“ vorliegt. Aufgrund seiner unterschiedlichen historischen Wurzeln sowie mehrerer gewohnheits- und richterrechtlich geprägter Institute, die nicht aufeinander abgestimmt sind, bildet das Recht der staatlichen Ersatzleistungen immer noch eine ,,mehrschichtige, lückenhafte und unübersichtliche Materie“; ${ }^{174}$ eine durchgreifende Reform ist nicht in Sicht, da das Staatshaftungsgesetz vom 26. Juni 1981 wegen fehlender Bundeskompetenz vom BVerfG für verfassungswidrig erklärt worden ist. ${ }^{175}$ Ossenbühls Anliegen war es von Anfang an nicht, ,„dem gewachsenen Chaos des Staatshaftungsrechts den Mantel der Rechtssystematik überzuwerfen“. Seine Darstellung geht vielmehr von ,einzelnen gewachsenen Anspruchsinstituten aus, stellt ihren Ursprung dar und verfolgt sodann ihre Entwicklung, insbesondere ihre Ausweitungen und ihr Hineinwachsen in den Anwendungsbereich der vorhandenen Anspruchsinstitute“. ${ }^{176}$ Pragmatisches Ziel ist die Vermittlung der

\footnotetext{
169 Forsthoff, Lehrbuch (Fn. 160), S. 48; 10. Aufl. 1974, S. 57.

170 Forsthoff, Lehrbuch (Fn. 160), S. 263 ff., 295; 10. Aufl. 1974, S. $368 \mathrm{ff} ., 410,567 \mathrm{ff}$.

171 Bumke, Methodik (Fn. 3), S. 92 f. Eingehend jetzt Schütte, Verwaltungsrechtswissenschaft (Fn. 167).

172 Eine Ausnahme bildet hier z.B. zur Vorschlag der Einführung der Gefährdungshaftung im Staatshaftungsrecht, vgl. Forsthoff, Lehrbuch (Fn. 160), S. $359 \mathrm{ff}$.

173 Neben dem Lehrbuch von Jellinek wurde das Lehrbuch von Forsthoff in den veröffentlichten Urteilen des BVerw $G$ in den ersten zwei Jahrzehnten der Bundesrepublik am weitaus häufigsten zitiert, vgl. die Analyse von Schulze-Fielitz, Verwaltungsrechtswissenschaft (Fn. 3), 435, Fn. 63. Der Einfluß auf die Rechtsprechung wird etwa auch hervorgehoben in den Besprechungen der 2. Auflage durch Bachof (JZ 1952, S. 606) und Schneider (NJW 1952, S. 972).

174 Mauer, Allgemeines Verwaltungsrecht, 16. Aufl. 2006, § 25, Rn. 1.

175 BVerfGE 61, $149 \mathrm{ff}$.

176 Ossenbühl, Staatshaftungsrecht, 5. Aufl. 1998, S. 2 f.
} 
Rechtspraxis und ihrer Hintergründe. ${ }^{177}$ Statt um Reform geht es um Selbstvergewisserung und Konsolidierung.

\section{Orientierung im Studium und Rechtsalltag}

a) Zwei zentrale Referenzwerke

Die Orientierung und Wegbegleitung im Studium und Rechtsalltag ist im Laufe der letzten fünf Jahrzehnte immer wichtiger geworden. Im Jahre 2005 hat man die Qual der Wahl zwischen über 30 Lehrbüchern zum Allgemeinen Verwaltungsrecht und über 15 Lehrbüchern zum Verwaltungsprozeßrecht. Zwei Lehrbücher verdienen wegen ihrer didaktischen Vorbildfunktion und ihrer viele Jahre lang unangefochtenen Spitzenstellung auf dem Lehrbuchmarkt besonderer Erwähnung: das „Verwaltungsprozeßrecht“ von Carl Hermann Ule und das „Allgemeine Verwaltungsrecht" von Hartmut Maurer, beide vom Beck-Verlag betreut.

Lange Zeit ohne Konkurrenz ${ }^{178}$ blieb das in der Reihe ,Juristische Kurz-Lehrbücher" zeitgleich zum Inkrafttreten der Verwaltungsgerichtsordnung erschienene Werk von Ule (1. Aufl. 1960, 9. Aufl. 1987), das als erste umfassende Lehrbuchdarstellung des neuen Verwaltungsprozeßrechts Generationen von Juristen geprägt hat. Obwohl in der erster Linie für Studierende und Referendare geschrieben, setzte sich auch die Praxis mit den Ausführungen von Ule intensiv auseinander, dem es nicht nur um Vermittlung des bestehenden Stoffes ging, sondern zugleich um systematische Fortentwicklung eines rechtsstaatlichen Verwaltungsprozeßrechts. ${ }^{179}$ Beispielhaft sei hier nur auf die von ihm begründete Vertretbarkeitslehre zur gerichtlichen Überprüfung unbestimmter Rechtsbegriffe ${ }^{180}$ hingewiesen, die sich letztlich freilich nicht durchgesetzt hat. ${ }^{181}$

Eine Lücke füllte auch das in der kompakten Reihe „Grundrisse des Rechts“ 1980 erstmals erschienene Lehrbuch von Maurer. ${ }^{182}$ Damals wie heute wendete es

177 Deutlich auch das Vorwort der 1. Aufl. aus dem Jahre 1976: „Die Bemerkung schließt ein, daß die Darstellung im wesentlichen den Stand der Rechtsprechung aufzeichnet und kritisch würdigt, jedoch auf die Ausbreitung solcher Haftungskonzeptionen verzichtet, die in der Theorie mit viel Scharfsinn entworfen worden sind, aber keine praktische Verwirklichung gefunden haben".

178 Im Jahre 1970 erschien der Grundriß des Verwaltungsprozeßrechts von Oskar Tschira und Walter Schmitt Glaeser, heute fortgesetzt als Schmitt Glaeser/Horn, Verwaltungsprozeßrecht (15. Aufl. 2000). 1972 erschien Stern, Verwaltungsprozessuale Probleme in der öffentlichrechtlichen Klausur (heute: Verwaltungsprozessuale Probleme in der öffentlich-rechtlichen Arbeit, 8. Aufl. 2000).

179 Deutlich hervorgehoben wird dieser Umstand etwa von Imboden in einer Besprechung der 2. Aufl. (1961), DVB1. 1961, S. 644.

180 Ule, Verwaltungsprozeßrecht, 9. Aufl. 1987, S. 7-16.

181 Eingehend dazu statt vieler Schmidt-Aßmann, in: Maunz/Dürig, GG, Art. 19 Abs. 4, Rn. 188-217a.

182 Die im Jahre 1980 ansonsten auf dem Markt befindlichen Lehrbücher zum Allgemeinen Verwaltungsrecht wiesen alle mehr oder weniger gravierende Schwächen auf: Götz, Allgemeines Verwaltungsrecht, 2. Aufl. 1979; Franz Mayer, Allgemeines Verwaltungsrecht, 4. Aufl. 1977; Obermayer, Grundzüge des Verwaltungsrechts und Verwaltungsprozeßrechts, 2. Aufl. 1975; Püttner, Allgemeines Verwaltungsrecht, 5. Aufl. 1979; Roellecke, Grundbegriffe des Verwaltungsrechts, 1972; Schweickhardt (Hrsg.), Allgemeines Verwaltungsrecht, 1979; Wallerath, Allgemeines Verwaltungsrecht, 1979. 
sich an jenen Studenten, den das Format des „Wolff/Bachof“ ebenso überforderte wie das große Lehrbuch von Forsthoff, der in den letzten Auflagen zudem neuere rechtliche Entwicklungen nur noch selektiv in den Fußnoten berücksichtigte. ${ }^{183}$ In einer klaren, unprätentiösen Sprache und didaktisch hervorragend aufbereitet präsentiert Maurer zuverlässig und übersichtlich den aktuellen Stand der Verwaltungsrechtsdogmatik unter Verarbeitung der einschlägigen Rechtsprechung und Literatur, nicht ohne immer wieder auch eigene Wertungen vorsichtig in die Darstellung einfließen zu lassen. ${ }^{184}$ Eine gewisse Zurückhaltung gegenüber neueren Entwicklungen bleibt dabei unverkennbar. Das zeigt sich etwa an der bis heute eher blaß bleibenden Entfaltung des Verfahrensgedankens, ${ }^{185}$ der erst späten Beachtung informeller und kooperativer Handlungsweisen der Verwaltung ${ }^{186}$ oder der zögerlichen Rezeption des Europarechts. ${ }^{187}$ Zentrale aktuelle Themenkomplexe wie z.B. die Privatisierung von Verwaltungsaufgaben oder die Informatisierung des Verwaltungshandelns ${ }^{188}$ finden sogar bis heute überhaupt keine oder nur sehr rudimentäre Berücksichtigung. ${ }^{189}$

\section{b) Größere Gesamtdarstellungen}

Wer als Student oder Praktiker an den über Kommentare nur schwer erschließbaren übergreifenden Strukturen des Verwaltungs- und Verwaltungsprozeßrechts interessiert war, dabei aber gleichzeitig auf Details und die vertiefte Erörterung von Einzelfragen nicht verzichten wollte, der war auf größere Gesamtdarstellungen mittleren Umfangs angewiesen. Das Angebot blieb hier lange Zeit überschaubar. Als solide Begleiter etablierten sich neben dem bereits erwähnten „Kurzhandbuch" von Hans J. Wolff und Otto Bachof der von Hans-Uwe Erichsen herausgegebene Sammelband zum „Allgemeinen Verwaltungsrecht“, an dem seit der 1. Auflage im Jahre 1975 einige der renommiertesten Verwaltungsrechtswissenschaftler mitwirken, ${ }^{190}$ das große, kommentarähnliche Lehrbuch von Carl Hermann Ule und Hans-Werner Laubinger zum ,Verwaltungsverfahrensrecht“, deren eingehende Erläuterung des neuen Verwaltungsverfahrensgesetzes Maßstäbe setzte (1. Aufl. 1977, 4. Aufl. 1995), sowie das von Rainer Pietzner und Michael Ronellenfitsch verfaßte Kompendium „Das Assessorexamen im Öffentlichen Recht: Widerspruchsverfahren und Verwaltungsprozeß“ (1. Aufl. 1977, 11. Aufl. 2005), dessen Zielgruppe Rechtsreferendare und Praktiker sind. Allen Werken gemeinsam war und ist das

183 Deutlich Worte findet der Vorsitzende Richter am BVerwG Hugo Kellner in einer Besprechung der 10. Auflage (NJW 1974, S. 1549): „So sind die Ausführungen (S. 85 ff.) zum Problemkreis ,unbestimmter Rechtsbegriff“ ... kaum geeignet, dem Leser auch nur eine ungefähre Vorstellung der Rechtsprechung und des Meinungsstandes zu vermitteln“.

184 Vgl. z. B. Maurer, Allgemeines Verwaltungsrecht (Fn. 174), § 4, Rn. 56, § 6, Rn. 11 b, 14, § 7, Rn. 34, 55 ff., § 8, Rn. 24f., § 9, Rn. 63b, § 12, Rn. 25 f., § 14, 24 f.

185 Maurer, Allgemeines Verwaltungsrecht (Fn. 174), § 19.

186 Jetzt Maurer, Allgemeines Verwaltungsrecht (Fn. 174), § 15, Rn. 14-21.

$187 \mathrm{Vgl}$. Maurer, Allgemeines Verwaltungsrecht (Fn. 174), § 2, Rn. 31-36.

188 Vgl. oben B I. 5.

189 Vgl. aber den kurzen Abschnitt zur „Verwaltungsautomation“ ( $\$ 18)$ und die einführenden

Darlegungen zu Formen der Privatisierung unter $\$ 23$, Rn. 60 ff.

190 Jetzt Erichsen/Ehlers (Hrsg.), Allgemeines Verwaltungsrecht, 13. Aufl. 2006. 
Bedürfnis nach Einpassung der stetig anschwellenden Stoffmenge in das von Otto Mayer entworfene normative Grundgerüst und seine weitere Verfeinerung. Diese Vorgehensweise ist letztlich mitverantwortlich für den hohen Ausdifferenzierungsgrad der deutschen Verwaltungsrechtsdogmatik, der nicht nur Bewunderung hervorruft, sondern auch immer wieder kritisiert wird, ,da zu viele feinziselierte Abgrenzungen wegen ihrer häufig mangelnden Trennschärfe in der praktischen Anwendung mehr Probleme aufwerfen als sie lösen“. ${ }^{191}$ Als besonders anschauliches Beispiel mag der Verwaltungsakt dienen, der sich vom einst ruhenden Pol in der ,flutenden Masse der Verwaltungstätigkeit“ (Otto Mayer) zu einem konstruktiven Monstrum entwickelt hat. Bezeichnungen wie relativer, konsentierter, interner, vorläufiger, allgemeinverfügender oder formaler Verwaltungsakt sowie die mit großer Akribie betriebene Qualifizierung von akzessorischen Nebenbestimmungen geben davon Zeugnis. ${ }^{192}$ Schon im Jahre 1958 konstatierte Werner Weber ${ }^{193}$ weitsichtig: „Die Jurisprudenz unserer Tage hat sich ... allzu stark in die Betrieblichkeit und Betriebsamkeit eines Perfektionismus der Spezialisierung mit hineinziehen lassen. Man spricht davon, daß das Leben so unerhört kompliziert geworden sei und daß Recht und Rechtswissenschaft dem folgen müßten. Jeder erachtet es für seine Pflicht, zu seinem Teile die Differenzierung kräftig weiterzutreiben, während doch der eigentliche Dienst des Rechts darin bestände, der Auffaserung des Lebens entgegenzuwirken." Nun wäre es freilich naiv zu meinen, eine komplizierte, unübersichtliche Welt ließe sich mit einfachen Mitteln beherrschen. Um den eingangs geschilderten Anforderungen an die Verwaltungsrechtsdogmatik angemessen Rechnung zu tragen, gilt es vielmehr den richtigen Mittelweg zu finden zwischen dem dogmatischen Anbau an Vorhandenes einerseits und konzeptioneller Neuorientierung andererseits.

\section{Irritationen, Impulse und Sonderwege}

Wenngleich die an der herkömmlichen Systematik (Rechtsquellen, Grundprinzipien, Handlungsformen, Verfahren, Organisation) ausgerichteten, im weitesten Sinne konventionellen Darstellungen des Verwaltungsrechts die Lehrbuchlandschaft in den ersten Jahrzehnten der Bundesrepublik prägten, fehlte es seit den 1980er Jahren doch nicht an Versuchen, neue Perspektiven auf den Stoff zu eröffnen, um auf diese Weise auch neue Impulse für die Fortentwicklung der Verwaltungsrechtsdogmatik zu gewinnen. Vier Werke verdienen hier besondere Erwähnung:

191 So Wahl, Entwicklungen des Fachplanungsrechts, NVwZ 1990, 426 (438), der deshalb für einen ,ökonomischen Gebrauch“ begrifflicher Unterscheidungen plädiert. Vgl. ferner Voßkuhle, Das Kompensationsprinzip, 1999, S. 89f. m.w. N.

$192 \mathrm{Zu}$ den unterschiedlichen Formen des Verwaltungsakts und seiner Entwicklung vgl. z. B. Ladeur, Die Zukunft des Verwaltungsakts - Kann eine Handlungsformenlehre aus dem Aufstieg des ,,informalen Verwaltungshandelns“ lernen?, VerwArch 86 (1995), $511 \mathrm{ff}$.; Schoch, Der Verwaltungsakt zwischen Stabilität und Flexibilität, in: Hoffmann-Riem/Schmidt-Aßmann (Hrsg.), Innovation und Flexibilität des Verwaltungshandelns, 1994, S. 199 ff.; Henneke, 30 Jahre LVwG, 20 Jahre VwVfG - Stabilität und Flexibilität des Verwaltungshandelns, DÖV 1997, 768 ff. Krit. Renck, Für einen formalisierten Verwaltungsakt, in: FS für Franz Knöpfle, 1996, S. 291 ff.

193 Weber, Spannungen und Kräfte im Westdeutschen Verfassungssystem, 3. Aufl. 1970, S. 106. 
An die Stelle der Handlungsformenlehre als maßgebliches Bauprinzip der verwaltungsrechtlichen Systembildung tritt in Norbert Achterbergs Lehrbuch zum ,Allgemeinen Verwaltungsrecht“, das im Jahr 1982 erstmals erschien (2. Aufl. 1986), die Rechtsverhältnislehre. Statt an die einzelne Entscheidung wird hier an die Rechtsbeziehung zwischen den Akteuren im Sinne einer Gesamtbetrachtung des Lebenssachverhalts angeknüpft. Dogmatischen Niederschlag hat die Rechtsverhältnislehre etwa bei der Auslegung und Lückenschließung der einschlägigen Gesetzesbestimmungen zum Subventions-, Anstaltsnutzungs-, Überwachungs-, Beamtenrechts- oder Steuerschuldverhältnis gefunden, ansonsten kommt ihr aber lediglich heuristische und strukturierende Funktion zu. ${ }^{194}$

Heute noch äußerst modern mutet die ebenfalls im Jahre 1982 in der JuSSchriftenreihe bei Beck erschienene und mittlerweile weitgehend vergessene ,Einführung in die Probleme des Verwaltungsrechts" von Walter Schmidt an. ${ }^{195}$ Aus einem problemorientierten Blickwinkel arbeitet er konsequent den funktionalen Zusammenhang zwischen Verwaltungsformenlehre, Verwaltungsorganisation, Verwaltungsverfahrensrecht und Verwaltungsprozeßrecht heraus. Dabei werden verfassungsrechtliche Vorgaben und privatrechtliche Institute ebenso integrativ in die Überlegungen mit einbezogen wie Teile des Besonderen Verwaltungsrechts und rechtstatsächliche Erkenntnisse.

Dem „Entscheidungsbedarf der nachindustriellen Gesellschaft“ möchte Heiko Faber in seinem „Verwaltungsrecht“ (1. Aufl. 1987, 4. Aufl. 1995) durch das von ihm sogenannte „Recht der Infrastrukturverwaltung“ nachkommen. ${ }^{196}$ Trotz der Forderung nach einer eigenständigen Theorie des Verwaltungsrechts ${ }^{197}$ und einer eigenwilligen Gliederung bleiben seine Ausführungen aber doch weitgehend den herkömmlichen Instituten und Kategorien verpflichtet.

Nicht fehlen darf an dieser Stelle schließlich der Hinweis auf das im NomosVerlag erschienene „System des Verwaltungsrechts“ von Roman Loeser (Bd. I und Bd. II, 1994). ${ }^{198}$ In mitunter glanzvoll-eklektizistischer Manier gelingt es Loeser, verwaltungsrechtsdogmatische, theoretische, verwaltungswissenschaftliche und methodische Erkenntnisse mit praktischen Hinweisen zu verknüpfen.

Alle vier genannten Werke haben auf die Rechtspraxis indes wenig bis gar keinen Einfluß gehabt. Offensichtlich ist der konventionelle Bezugshorizont des praktisch arbeitenden und durch die juristische Methode geschulten Juristen für (produktive) Irritationen und Sonderwege nicht sonderlich empfänglich. ${ }^{199}$

\footnotetext{
${ }^{194}$ So zutreffend Schmidt-Aßmann, Ordnungsidee (Fn. 14), 6. Kap., Rn. 40-45.

195 Angemessene Würdigung z. B. bei Becker, BayVBl. 1983, S. 703.

${ }^{196}$ Faber, Verwaltungsrecht (Fn. 150), S. $350 \mathrm{ff}$.

197 Faber, Verwaltungsrecht (Fn. 150), S. $26 \mathrm{ff} ., 167 \mathrm{ff}$.

198 Band 1 behandelt die Allgemeinen Lehren, Methoden und Techniken des Verwaltungsrechts, Band 2 die Verwaltungsorganisation und Band 3, der von Buchholz verfaßt wurde und 1987 erschienen ist, die Handlungsformen und das Verwaltungsverfahren.

${ }^{199}$ Exemplarisch für die eher ablehnende Grundhaltung der Zunft sind die Besprechungen der Werke von Achterberg (DVBl. 1983, 1028) und Faber (DVBl. 1988, 915) durch Schröder.
} 


\section{Kommentare und Handbücher}

Die weitaus wichtigsten Arbeitsmittel für den deutschen Verwaltungsjuristen sind im Alltag Kommentare und Handbücher. Ihre Aufgabe ist es in erster Linie, den Meinungsstand zu einem bestimmten Rechtsproblem unter besonderer Berücksichtigung der Rechtsprechung umfassend und kompakt aufzubereiten. ${ }^{200}$ Dabei wird häufig übersehen, daß bereits die schlichte Darstellung der „Rechtslage“ von Vorverständnissen und Wertungen bestimmt ist. Gerade Erstkommentierungen können insoweit auch richtungsweisend sein.

\section{Zwei Pionierwerke der ersten Stunde}

a) Die Kommentierung der Verwaltungsgerichtsordnung durch Erich Eyermann und Ludwig Fröhler

Pünktlich zum Erlaß der Verwaltungsgerichtsordnung vom 21. Januar 1960 erschien im Beck-Verlag ihre erste Kommentierung von Erich Eyermann, damals Senatspräsident des Bayerischen Verwaltungsgerichtshofs, und Ludwig Fröhler, ebenfalls Mitglied des Bayerischen Verwaltungsgerichtshofs und zuletzt Professor an der Universität Linz. ${ }^{201}$ Das Werk erlebte kurz hintereinander neun Auflagen und fehlte lange Zeit auf kaum einem Verwaltungsrichterschreibtisch. 1998 erschien unter dem Namen „Eyermann“ eine vollständige Neubearbeitung, die aber noch denselben Geist atmet wie die Ursprungsfassung: Auch hier erläutern Praktiker nüchtern und übersichtlich die einschlägigen Normen und die dazu ergangenen gerichtlichen Entscheidungen; auf das wissenschaftliche Schrifttum wird überwiegend nur pauschal verwiesen, ohne die dort vorgebrachten einzelnen Argumente inhaltlich näher zu würdigen; es dominiert ein knapper, eher autoritativer Stil. ${ }^{202}$ Durch diese Form der Selbstkanonisierung verfestigte und verselbständigte sich die gerichtliche Praxis in der Folgezeit weiter. ${ }^{203}$

\section{b) Die Kommentierung des Verwaltungsverfahrensgesetzes des Bundes durch Ferdinand O. Kopp}

Vom Duktus des „Eyermann/Fröhler“ unterscheidet sich eine andere Pionierleistung auf dem Gebiet der Kommentarliteratur, was freilich auch der Materie geschuldet sein könnte. Als das Verwaltungsverfahrensgesetz am 1. Januar 1977 in Kraft trat, bestanden aufgrund seiner wechselvollen und langwierigen Entstehungsgeschichte, teilweise abweichender landesrechtlicher Regelungen sowie den

${ }^{200}$ Das schließt innovative wissenschaftliche Leistungen freilich nicht aus, vgl. auch SchulzeFielitz, JöR 50 n.F (2002), 1 (19f.) m.w. N.

${ }^{201}$ Beide Autoren hatten bereits die Verwaltungsgerichtsgesetze für Bayern, Bremen, Hessen und Württemberg-Baden kommentiert (1. Aufl. 1950, 2. Aufl. 1954).

202 So bereits der Senatspräsident des OVG Lüneburg, Walter Tietgen, in der Besprechung der 1. Aufl., DVB1. 1960, 867.

203 Zur nicht zu unterschätzenden Bedeutung der publizistischen Begleitung der eigenen Rechtsprechung durch die Richter des BVerwG aufschlußreich Schulze-Fielitz, Bundesverwaltungsgericht (Fn. 3), S. 1078 ff. m. w. N. 
bereits verfestigten gewohnheitsrechtlichen Grundlagen des Verwaltungsrechts nicht unerhebliche Unsicherheiten über die konkrete Auslegung und Reichweite der einzelnen Vorschriften. Besonders prädestiniert, hier eine Bresche durch das Dickicht zu schlagen, schien Ferdinand O. Kopp, nicht nur wegen seines bereits sehr gut von der Praxis aufgenommenen Kommentars zur Verwaltungsgerichtsordnung, der den Eyermann-Fröhler als maßgeblichen Handkommentar verdrängen sollte (1. Auflage 1973), sondern vor allem auch wegen seiner 1971 - ebenfalls im Beck-Verlag veröffentlichten Habilitationsschrift ,Verfassungsrecht und Verwaltungsverfahrensrecht“. Kopp setzte sich in seiner zupackenden und trotz des kompakten Formats durchaus eigenständigen, dogmatisch reflektierten und kritischen Kommentierung, ${ }^{204}$ die bereits 1976 auf den Markt kam, ${ }^{205}$ denn auch für eine rechtsschutzfreundliche Auslegung der einzelnen Verfahrensvorschriften ein, gut abzulesen etwa an den einleitenden Ausführungen zu $§ \S 45 f$. VwVfG. ${ }^{206}$ Die Rechtsprechung ist ihm in den meisten kontroversen Fragen nicht gefolgt; das allgemeine Bewußtsein für die zentrale rechtstaatliche Bedeutung des bis zu diesem Zeitpunkt eher vernachlässigten Verwaltungsverfahrens sollte durch seine Kommentierung, die er über sechs Auflagen betreute, ${ }^{207}$ jedoch insgesamt nachhaltig gestärkt werden.

\section{Die Reaktion auf die Ausdifferenzierung der Verwaltungs(prozeß)rechtsordnung: Großkommentare}

Auf Dauer boten die Kurzkommentare zu wenig Raum, um die Vielzahl der von der Rechtspraxis aufgeworfenen Rechtsfragen und die Flut von gerichtlichen Entscheidungen und Literatur umfassend darstellen und würdigen zu können. Es war aber nicht nur das Interesse am Detail und der erreichte Grad der Ausdifferenziertheit der Verwaltungs(prozeß)rechtsordnung, die die Verlage dazu ermutigten, Großkommentare verstärkt in ihr Sortiment aufzunehmen. Vielmehr zeigte sich, daß viele Kommentierungen der ersten Generation zur VwGO und zum VwVfG veraltet waren, neuere Entwicklungen wie die der Europäisierung nur zögernd und selektiv verarbeitet wurden und der Zeitpunkt gekommen war, den gesamten Rechtsstoff noch einmal neu zu sichten und zu ordnen. Teilweise begnügte man sich damit, neue Autoren für bereits vorhandene Werke zu verpflichten, die dann deutlich an Umfang zulegten, wie z.B. die Kommentierung von Eyermann und Fröhler ${ }^{208}$ oder Stelkens, Bonk und Sachs, ${ }^{209}$ teilweise entstanden völlig neue Werke, wie die von Helge Sodan und Jan Ziekow sowie Friedrich Schoch, Eberhard SchmidtAßmann und Rainer Pietzner herausgegebenen Kommentare zur VwGO, die in den Jahren 1995 und 1996 erschienen. Gerade das letzte Werk belegt die Leistungs-

204 Vgl. auch die positiven Besprechungen der 1. Aufl. des Kommentars von Grundei, NJW 1977, S. 712; Ule, DVBl. 1977, S. 398 und Renck, BayVBl. 1977, S. 479.

205 Parallel erschienen: Meyer/Borgs, Verwaltungsverfahrensgesetz, 1976; Knack (Hrsg.), Verwaltungsverfahrensgesetz, 1976. 1978 folgte Stelkens/Bonk/Leonhardt, Verwaltungsverfahrensgesetz.

206 Vgl. auch S. XLI ff. der Allgemeinen Vorbemerkung in der 1. Aufl. 1976.

207 Seit der 7. Auflage (2000) wird das Werk von Ramsauer fortgeführt (9. Aufl. 2005).

208 1. Aufl. 1960: 741 Seiten, 11. Aufl. 2000: 1516 Seiten.

209 1. Aufl. 1978: 858 Seiten; 6. Aufl. 2001: 2739 Seiten. 
fähigkeit der Literaturgattung „Kommentar“ für die Fortbildung des Rechts. Insbesondere in den abschnittsweise eingefügten Vorbemerkungen finden sich immer wieder weiterführende konzeptionelle Überlegungen, die auch von der Praxis rezipiert werden, so z.B. die Ausführungen zur Grundrechts- und Rechtssatzabhängigkeit des subjektiven öffentlichen Rechts von Rainer Wahl210 oder zur Funktionsidentität von aufschiebender Wirkung und einstweiliger Anordnung, in denen Schoch ${ }^{211}$ Erkenntnisse seiner Habilitationsschrift ${ }^{212}$ fruchtbar macht.

\section{Reform: Das Projekt der „Grundlagen des Verwaltungsrechts"}

Die eingangs konstatierten grundlegenden Veränderungen im Verwaltungsrecht finden in der Lehrbuch- und Kommentarliteratur bis heute kaum Berücksichtigung. Lediglich die breiter angelegten Monographien ${ }^{213}$ von Eberhard Schmidt-Aßmann (Das Allgemeine Verwaltungsrecht als Ordnungsidee, 1. Aufl. 1998, 2. Aufl. 2004) und Gunnar Folke Schuppert (Verwaltungsrechtswissenschaft, 2000) können für sich in Anspruch nehmen, die von ihnen selbst maßgeblich mit angestoßenen Entwicklungen methodisch und inhaltlich aufzunehmen und fortzuführen. Eine Zäsur bildet insofern das vom Beck-Verlag betreute Projekt „Grundlagen des Verwaltungsrechts“. Unter der Ägide der Herausgeber Wolfgang Hoffmann-Riem, Eberhard Schmidt-Aßmann und Andreas Voßkuhle sind hier über 50 reformorientierte Verwaltungsrechtswissenschaftler bestrebt, gemeinsam - begleitet durch zahlreiche Workshops - die Summe jener wissenschaftlichen Bemühungen zu ziehen, die während der letzten Jahrzehnte im Verwaltungsrecht stattgefunden haben, um auf diesem Fundament einen wesentlichen Beitrag zu seiner Fortentwicklung zu leisten. Der erste von drei Bänden erschien im Herbst des Jahres 2006.

\section{Folgerungen für das Verhältnis von Rechtswissenschaft und Rechtspraxis}

Insgesamt gesehen wird man den Einfluß der Lehrbuch- und Kommentarliteratur auf die Entwicklung des Allgemeinen Verwaltungs- und Verwaltungsprozeßrechts in den letzten sechs Jahrzehnten nach alledem eher als gering veranschlagen müssen. ${ }^{214}$ Sie , arbeitete der Rechtsprechung vor und nach, sie umspielte sie gewissermaßen, konnte aber selbst nichts Verbindliches mehr dazu beitragen. "215 Im

${ }^{210}$ Wahl, in: Schoch/Schmidt-Aßmann/Pietzner (Hrsg.), VwGO, Vorb § 42, Rn. 75-117.

211 Schoch, in: ders./Schmidt-Aßmann/Pietzner (Hrsg.), VwGO, Vorb § 80, Rn. 45-50.

212 Schoch, Vorläufiger Rechtsschutz und Risikoverteilung im Verwaltungsrecht, 1988.

213 Es handelt sich bei beiden Werken nicht um Lehrbücher im klassischen Sinne, vgl. hierzu die Besprechungen von Schulze-Fielitz, JZ 1998, $1163 \mathrm{ff}$, und Voßkuhle, Der Staat 41 (2002), $487 \mathrm{ff}$.

214 So auch Schulze-Fielitz, Bundesverwaltungsgericht (Fn. 3), S. 1061 (1074); ders., Verwaltungsrechtswissenschaft (Fn. 3), $421 \mathrm{ff}$. Allgemein zum Theorie-Praxis-Bruch vgl. zuletzt die Beiträge in: Krawietz/Morlok (Hrsg.), Vom Scheitern und der Wiederbelebung juristischer Methodik im Rechtsalltag - ein Bruch zwischen Theorie und Praxis?, 2002, und in: Engel/Héritier (Hrsg.), Linking Politics and Law, 2003.

215 Stolleis, Verwaltungsrechtswissenschaft (Fn. 3), S. 243, zur Rolle der Verwaltungsrechtswissenschaft insgesamt. Vgl. ferner Schulze-Fielitz, Bundesverwaltungsgericht (Fn. 3), S. 1071: „Das Gericht geht voran, die begleitende Wissenschaft trägt die Schleppe“. 
Vordergrund standen Konsolidierung, Vermittlung und Fortschreibung des Bestehenden. Neue Ansätze setzten sich, soweit überhaupt vorhanden, nur sehr selten durch. Über die Ursachen dieses Befundes läßt sich an dieser Stelle nur spekulieren. Fünf Punkte erscheinen mir wesentlich:

(1) Zum einen wohnt dem durch die weiterhin dominante juristische Methode geprägten Selbstverständnis des Verwaltungsrechtswissenschaftlers von Haus aus ein beharrendes Moment inne. Man denkt nicht vom Problem her, sondern beschäftigt sich mit vorgefundenen Rechtsakten, die in Beziehung zueinander gesetzt werden. Aufgrund der daraus resultierenden Theorieferne und mangelnder Kenntnis der Realbereiche fehlt es in der Regel an Maßstäben und Legitimation für im weitesten Sinne rechtspolitische Vorschläge, die zudem schnell mit dem Hautgout des Unwissenschaftlichen versehen werden.

(2) Umgekehrt hat die rechtsschutzfreundliche Ausrichtung des Grundgesetzes ein Primat der Rechtsprechung errichtet. Da zu den meisten Fragen des Verwaltungs- und Verwaltungsprozeßrechts vergleichsweise schnell verwaltungsgerichtliche Entscheidungen vorliegen, befinden sich Gesetzgeber und Wissenschaft permanent in der Defensive; meistens müssen sie auf bereits Vorgefundenes reagieren, votieren dabei aber ungern gegen die Praxis.

(3) Ferner kommen die maßgeblichen Impulse für Innovationen mehr denn je aus den Referenzgebieten des Besonderen Verwaltungsrechts. ${ }^{216}$ Dort wird das Anschauungsmaterial gewonnen für mögliche Reformen; dort findet die Erprobung neuer Institute, Prinzipien und Regelungsarrangements statt, dort sind Gesetzgebung und Rechtsprechung besonders sensibel und aufnahmebereit für Anregungen aus der Wissenschaft; ${ }^{217}$ der wissenschaftliche Transfer in das Allgemeine Verwaltungsrecht erfolgt häufig zeitversetzt und lediglich nachvollziehend. ${ }^{218}$

(4) Bei alledem darf man auch ökonomische Aspekte nicht vernachlässigen: Verlage wollen und müssen mit ihren Produkten Geld verdienen; dieser Umstand färbt offensichtlich auch auf potentielle Autoren ab. Größere Auflagen versprechen in der Regel nur dann kommerziellen Erfolg, wenn sie sich rigoros an den Bedürfnissen der Ausbildung und der Praxis ausrichten; in beiden Bereichen ist die Nachfrage nach zukunftsgerichteter Reflexion aber (verständlicherweise) eher gering ausgeprägt. ${ }^{219} \mathrm{Im}$ Vordergrund stehen kompakte, leicht zugängliche Nachschlagewerke und Lernmaterialien.

216 Zur Bedeutung der Referenzgebiete für die Fortentwicklung des Verwaltungsrechts vgl. nur Schmidt-Aßmann, Ordnungsidee (Fn. 14), 1. Kap., Rn. 12 ff., 3. Kap., Rn. 2 ff.

217 Als Beispiel sei hier nur auf die breite Rezeption der 1. Auflage des Lehrbuchs von Kloepfer zum Umweltrecht (1. Aufl. 1989) verwiesen.

${ }^{218} \mathrm{Zu}$ den Novellierungen des Verwaltungsverfahrensgesetzes (vgl. oben B. I. 3.) findet sich z.B. im Vorfeld kaum ein Vorschlag oder Hinweis in der Lehrbuch- und Kommentarliteratur zum Allgemeinen Verwaltungsrecht.

${ }^{219}$ Krit. zur derzeitigen Lehrbuchkultur im Öffentlichen Recht Schulze-Fielitz, JöR n. F. 50 (2002), 1 (23); Möllers/Voßkuhle, Die deutsche Staatsrechtswissenschaft im Zusammenhang der internationalisierten Wissenschaften, DV 36 (2003), 321 (325f.); speziell zum Verwaltungsrecht vgl. Stolleis, Verwaltungsrechtswissenschaft (Fn. 3), 254f.; Brohm (Fn. 17), in: FS für Hartmut Maurer, 2001, S. 1079 (1081). 
(5) Folglich bleibt die Aufgabe, neue Impulse zu setzen und rechtliche Innovationen vorzubereiten, heute im wesentlichen den wissenschaftlichen Literaturgattungen „Monographie“ und „Aufsatz“ vorbehalten. Ihre breite Rezeption durch eine zunehmend unter dem Diktat von Zeit- und Ressourcenknappheit agierende Praxis stößt aber immer schneller an Grenzen. ${ }^{220}$ Diesen Umstand mag man bedauern, in gewisser Weise ist er aber auch der Preis für die im internationalen Vergleich wohl einmalig enge Verbindung zwischen der Rechtswissenschaft und der Rechtspraxis in der Bundesrepublik.

${ }^{220}$ Vgl. auch Redeker, Pflichtlektüre?, NJW 1976, 2200 ff. Krit. zum (vermeintlichen) „Zitierdefizit" in gerichtlichen Entscheidungen dagegen z. B. Erichsen, Das Bundesverwaltungsgericht und die Verwaltungsrechtswissenschaft, DVBl. 1978, 569 (571f.). 